\title{
A POSTERIORI ANALYSIS FOR SPACE-TIME, DISCONTINUOUS IN TIME GALERKIN APPROXIMATIONS FOR PARABOLIC EQUATIONS IN A VARIABLE DOMAIN
}

\author{
D.C. ANTONOPOULOU ${ }^{\$ *}$, M. PLEXOUSAKIS ${ }^{\dagger *}$
}

\begin{abstract}
This paper presents an a posteriori error analysis for the discontinuous in time space-time scheme proposed by Jamet for the heat equation in multi-dimensional, non-cylindrical domains 25]. Using a Clément-type interpolant, we prove abstract a posteriori error bounds for the numerical error. Furthermore, in the case of two-dimensional spatial domains we transform the problem into an equivalent one, of parabolic type, with space-time dependent coefficients but posed on a cylindrical domain. We formulate a discontinuous in time space-time scheme and prove a posteriori error bounds of optimal order. The a priori estimates of [19] for general parabolic initial and boundary value problems are used in the derivation of the upper bound. Our lower bound coincides with that of Picasso [36], proposed for adaptive, RungeKutta finite element methods for linear parabolic problems. Our theoretical results are verified by numerical experiments.
\end{abstract}

Keywords: Heat equation, space-time discontinuous Galerkin methods, a posteriori error estimates, noncylindrical domains.

\section{INTRODUCTION}

1.1. The problem. The discontinuous Galerkin method was introduced by Reed and Hill in 1973 37 and Lesaint and Raviart in 1974 29. This method has been proven to be efficient when applied to problems posed on domains of complicated geometry, approximates well discontinuous solutions and can be combined effectively with refinement or adaptivity techniques; see for instance [17, 12, 13, 14. Jamet in 25] considered the discontinuous Galerkin method for parabolic problems in general space-time finite element spaces.

The problem considered in this paper is posed on a domain of variable geometry in time and thus involves a moving boundary. We refer to the classical results of Baines and Miller in [9, 34, on the development of the so-called moving finite elements for the numerical approximation of a wide class of PDEs of hyperbolic and parabolic type. Makridakis and Nochetto in 31 presented an a posteriori error analysis for high order discontinuous Galerkin numerical schemes for evolutionary problems. In [2, issues such as optimality of estimates, adaptivity and nodal convergence rates were investigated, while in [26] a discontinuous Galerkin finite element method was applied to the non-linear Schrödinger equation in cylindrical domains. Picasso in [36] analyzed Runge-Kutta, adaptive finite element methods for linear parabolic problems.

More recently, in [7, the authors presented an a priori error analysis for a method analogous to (1.3) for the linear Schrödinger equation in non-cylindrical domains; see also the interesting work of Schötzau and Wihler in 38 . for the a posteriori error analysis of hp-methods for the time discretization of parabolic problems with continuous and discontinuous Galerkin methods.

The numerical scheme proposed in this work is a genuine space-time finite element method. Recently, there has been a renewed interest on high order space-time schemes due to their effectiveness when applied, for example, to problems in fluid dynamics, elasticity or conservation laws, even on unstructured grids. We refer to the works of van der Vegt and van der Ven on inviscid compressible flows [41, 42]; see also in [28] for

1991 Mathematics Subject Classification. 65M12,65M15,65M60.

${ }^{\dagger}$ Department of Mathematics and Applied Mathematics, University of Crete, GR-714 09 Heraklion, Greece.

\$ Department of Mathematics, University of Chester, Thornton Science Park, CH2 4NU, UK.

* Institute of Applied and Computational Mathematics, FORTH, GR-711 10 Heraklion, Greece. 
the case of the compressible Navier-Stokes equations, and the recent results of Dumbser and Tavelli for threedimensional Navier-Stokes equations and linear elasticity for finite elements on unstructured meshes [39, 40]. May and Zakerzadeh [32] proved convergence of space-time discontinuous Galerkin approximations for scalar hyperbolic conservation laws by avoiding the so-called streamline-diffusion stabilization. Hiltebrand, Mishra and Parés constructed entropy-stable approximations for non-conservative hyperbolic systems [22, while in 21, the authors considered multi-dimensional nonlinear systems of conservation laws.

We consider a bounded domain $\Omega(t)$ in $R^{m}, m \geq 1$, continuously dependent on $t \in[0, T]$. We let $\Gamma(t)$ denote the boundary of $\Omega(t)$ and

$$
S_{T}:=\{(x, t): x \in \Omega(t), 0<t<T\}, \quad \Sigma_{T}:=\{(x, t): x \in \Gamma(t), 0<t<T\} .
$$

We seek $u: \bar{S}_{T} \rightarrow R$ such that

$$
\begin{aligned}
& u_{t}=\Delta u+f \text { in } S_{T}, \\
& u=0 \text { in } \Sigma_{T}, \\
& u=u^{0} \quad \text { in } \Omega(0),
\end{aligned}
$$

where $f$ is a function defined on $S_{T}$ and $u^{0}$ on $\Omega(0)$. Detailed a priori error analysis of discontinuous in time space-time schemes for this problem has been carried out by Jamet 25]. We note that (1.1) is posed on a domain varying in time, so it may be thought of as a Stefan type problem. However, the boundary $\Gamma(t)$ does not depend on the solution. Hence, it is not a free boundary problem.

We employ standard notation for the Sobolev spaces used throughout. For $S$ a subdomain of $S_{T}$, we denote by $\widetilde{H}^{1}(S)$ the space of functions in $H^{1}(S)$ that vanish on $\Sigma_{T} \cap \bar{S}$. We let $((\cdot, \cdot))_{S}$ denote the inner product and $\|\cdot\|_{S}$ the corresponding norm in $L^{2}(S)$. Similarly, we denote by $(\cdot, \cdot)_{\Omega(t)}$ the inner product in $L^{2}(\Omega(t))$ and by $|\cdot|_{\Omega(t)}$ the corresponding norm.

For $0=t^{0}<t^{1}<\cdots<t^{N}=T$, a partition of $[0, T]$, we write $\Omega^{n}:=\Omega\left(t^{n}\right)$ and

$$
G^{n}:=\left\{(x, t): x \in \Omega(t), t \in\left(t^{n}, t^{n+1}\right)\right\}, \quad \widetilde{G}^{n}:=\left\{(x, t): x \in \bar{\Omega}(t), t \in\left(t^{n}, t^{n+1}\right]\right\} .
$$

Also, for $0 \leq \tau_{0}<\tau_{1} \leq T$, we let

$$
G\left(\tau_{0}, \tau_{1}\right)=\left\{(x, t): x \in \Omega(t), \tau_{0}<t<\tau_{1}\right\} .
$$

For each $0 \leq n \leq N-1$ we consider a family $\left\{V_{h}^{n}\right\}$ of finite dimensional subspaces of $\widetilde{H}^{1}\left(G^{n}\right)$ parametrized by $0<h \leq 1$. $V_{h}$ will denote the space of all functions defined on $\bar{S}_{T}$ such that their restriction on each $\widetilde{G}^{n}$ coincides with the restriction to $\widetilde{G}^{n}$ of a function $v_{h} \in V_{h}^{n}$. Functions in $V_{h}$ are, in general, discontinuous at the temporal nodes $t^{n}$. Consequently, we shall use the notation introduced in [25, and also used in 4, 7,

$$
v_{h}^{n}(\cdot):=v_{h}\left(\cdot, t^{n}\right) \quad \text { for } 0 \leq n \leq N,
$$

and

$$
v_{h}^{n+0}(\cdot):=\lim _{\epsilon \rightarrow 0^{+}} v_{h}\left(\cdot, t^{n}+\epsilon\right) \quad \text { for } 0 \leq n \leq N-1 .
$$

Here, note that $v_{h}\left(\cdot, t^{n}\right)=\lim _{\epsilon \rightarrow 0^{+}} v_{h}\left(\cdot, t^{n}-\epsilon\right)$.

In the analysis below we shall employ a transformation of the form $u \rightarrow e^{c t} u$, with $c$ a suitable constant, and consider the initial and boundary value problem

$$
\begin{aligned}
& u_{t}=\Delta u+\beta u+f \text { in } S_{T}, \\
& u=0 \text { in } \Sigma_{T}, \\
& u=u^{0} \quad \text { in } \Omega(0),
\end{aligned}
$$

where $\beta$ is a suitably small, negative constant.

Remark 1.1. The necessity of this transformation will be analyzed in the sequel. We shall show that it enables us to, for example, use an $L^{2}$-stability argument in the proof of the upper bound (2.7), and derive the a posteriori $L^{2}$ norm estimate (2.23) for the initial problem posed on the non-cylindrical domain. Moreover, 
when our scheme is applied to the equivalent continuous problem, a second order parabolic equation posed on a cylindrical domain, with space-time dependent coefficients, we prove existence of the numerical solution by $L^{2}$-stability, cf. Theorem 3.1, and derive an $H^{1}$ estimate for the a posteriori error. The exponential transformation $u \rightarrow e^{c t} u$ was introduced by Antonopoulou in [4] and has been used in [5, 7, 6] for the linear Schrödinger equation.

1.2. The numerical scheme: The discontinuous Galerkin method for $\sqrt{1.2}$ is defined as follows: Find $u_{h} \in V_{h}$ satisfying

$$
\begin{aligned}
B_{n}\left(u_{h}, v_{h}\right) & =\left(\left(f, v_{h}\right)\right)_{G^{n}}, \quad \forall v_{h} \in V_{h}^{n}, n=0, \ldots, N-1, \\
u_{h}^{0} & =u^{0},
\end{aligned}
$$

where the bilinear form $B_{n}\left(u_{h}, v_{h}\right)$ is given by

$$
\begin{aligned}
B_{n}\left(u_{h}, v_{h}\right):= & -\left(\left(u_{h}, \partial_{t} v_{h}\right)\right)_{G^{n}}+\left(\left(\nabla u_{h}, \nabla v_{h}\right)\right)_{G^{n}} \\
& +\left(u_{h}^{n+1}, v_{h}^{n+1}\right)_{\Omega^{n+1}}-\left(u_{h}^{n}, v_{h}^{n+0}\right)_{\Omega^{n}}-\left(\left(\beta u_{h}, v_{h}\right)\right)_{G^{n}} .
\end{aligned}
$$

Existence and a priori estimates for finite element spaces of general type for the proposed numerical scheme (1.4) have been proved by Jamet in [25] for $\beta=0$. The same results easily extend for nonzero $\beta$.

1.3. Main results and estimation strategy. In this work we undertake the a posteriori error analysis of the classical space-time numerical scheme of Jamet (25]) proposed for parabolic problems posed on noncylindrical domains. In Section 2, we present an a posteriori error analysis for problem 1.2 . We derive upper bounds for the error by utilizing the Clément type interpolant of Bernardi [11] in the multi-dimensional case. In the analysis we assume a sufficiently smooth space-time boundary and use local trace estimates. Our finite element space consists of piecewise linear functions in the $t$ and $x$ variables. Since $\operatorname{dim}(\Omega(t))=m$, the inner elements of our partitions consist of $m+1$-simplices, while the boundary elements are assumed compatible with a possibly curved boundary, [11.

In Section 3, we analyze the two-dimensional case of the spatial variables and transform our problem into an equivalent one posed on a parallelepiped of $\mathbb{R}^{3}$ (1-dimension for time and 2-dimensions for space). The new problem is of the general parabolic form, with space-time dependent coefficients. Since the transformed domain is cylindrical, space-time integration is commutative. This enables the coupling of the local $L^{2}$ projection in time with the standard 2-dimensional Clément interpolant in space, [16. The interpolation error is estimated in various norms. This is achieved by using certain local trace and Sobolev inequalities together with the well known space-time $a$ priori high-order estimates of the continuous problem presented in [19. As a result, we derive an a posteriori upper bound of optimal order. In addition, following a method proposed by Picasso in [36] we prove a lower bound of optimal order.

In Section 4 , considering the case $m=2$, i.e., when the spatial domain is in $\mathbb{R}^{2}$, we implement the proposed scheme in a FORTRAN/C++ code. The experimental results verify the optimal order of convergence. Furthermore, the a posteriori upper bound constants are approximated numerically. Their computed values are small, fact that motivates strongly the use of the proposed estimator in applications.

Finally, in the Appendix, we present some trace and Sobolev inequalities that we use throughout our analysis. Here, we focus on the dependence of the estimates on the diameter of the domain, so as to apply them locally for each element of the partition. In this case the bounds will involve the discretization parameters. We note, also, that the finite element partitions considered in this work are assumed to be regular, uniformly on $n$, but we do not impose any other restriction on the meshes. Two main ideas, related to the derivation of the a posteriori upper bounds, are presented and implemented in this work:

1. The use of the regularity of the continuous problem. The error $\varepsilon:=u-u_{h}$ of the numerical scheme is the difference between the continuous and the discrete solution. In order to derive upper bounds, since our method uses a space-time finite element formulation, we construct an appropriate interpolant of $\varepsilon$ in space and time variables. As a result, high order derivatives of the error $\varepsilon$ appear in the upper bound, stemming from the interpolation error, which cannot be estimated directly. Restricting ourselves to each element 
D.C. ANTONOPOULOU ${ }^{\$ *}$, M. PLEXOUSAKIS ${ }^{\dagger *}$

of the partition, we estimate the derivatives of the exact solution by using the space-time a priori high order estimates of Evans [19] for the general parabolic initial and boundary value problems with space-time coefficients. Since the bound is a posteriori, the derivatives of the numerical solution may be used in the estimator.

2. We control the constants appearing in the estimator and the error by choosing sufficiently high order polynomial approximations in time. This choice affects only slightly the estimator's computational cost compared to the alternate choice of a high order approximation in space, which is multi-dimensional. This flexibility is seen as an advantage of our method since it allows its implementation without having to change the numerical scheme at each time interval; the degree of polynomial approximation in time can be independently chosen, as high as we wish, between two temporal nodes since the scheme is discontinuous in time, in contrast to, say, Runge-Kutta (RK) methods. Considering high order RK methods, we refer to the classical result of Alexander, who introduced the diagonally implicit RK methods (DIRK) for stiff ODEs, [3]; cf. also in [18, or in [35] for the application of DIRK schemes to hyperbolic systems.

\section{A POSTERIORI ERROR ANALYSIS ON THE INITIAL DOMAIN}

2.1. Basic identities. Let us define, for $x \in \Omega(t)$,

$$
u^{n}(x):=\lim _{\epsilon \rightarrow 0^{+}} u\left(x, t^{n}-\epsilon\right) \quad \text { for } \quad 0 \leq n \leq N,
$$

and

$$
u^{n+0}(x):=\lim _{\epsilon \rightarrow 0^{+}} u\left(x, t^{n}+\epsilon\right) \quad \text { for } \quad 0 \leq n \leq N-1,
$$

where $u$ is the solution of 1.2. Obviously, if $u$ is continuous on $t^{n}$, then $u^{n}(x)=u^{n+0}(x)=u\left(x, t^{n}\right)$. If $u \in L^{2}\left(G^{n}\right),\|\nabla u\|_{G^{n}}<\infty$, and $u \in L^{2}\left(\Omega^{n}\right) \cap L^{2}\left(\Omega^{n+1}\right)$, for any $0 \leq n \leq N-1$, then for any $v_{h} \in V_{h}$ the following equality holds true

$$
\begin{aligned}
\left(\left(f, v_{h}\right)\right)_{G^{n}}=- & \left(\left(u, \partial_{t} v_{h}\right)\right)_{G^{n}}+\left(\left(\nabla u, \nabla v_{h}\right)\right)_{G^{n}} \\
& +\left(u, v_{h}^{n+1}\right)_{\Omega^{n+1}}-\left(u, v_{h}^{n+0}\right)_{\Omega^{n}}-\left(\left(\beta u, v_{h}\right)\right)_{G^{n}} \\
=- & \left(\left(u, \partial_{t} v_{h}\right)\right)_{G^{n}}+\left(\left(\nabla u, \nabla v_{h}\right)\right)_{G^{n}} \\
& +\left(u^{n+1}, v_{h}^{n+1}\right)_{\Omega^{n+1}}-\left(u^{n+0}, v_{h}^{n+0}\right)_{\Omega^{n}}-\left(\left(\beta u, v_{h}\right)\right)_{G^{n}},
\end{aligned}
$$

for any $0 \leq n \leq N-1$. We define the error $\varepsilon(x, t):=u(x, t)-u_{h}(x)$, and set

$$
\varepsilon^{n}(x):=\lim _{\epsilon \rightarrow 0^{+}} u\left(x, t^{n}-\epsilon\right)-u_{h}^{n}(x), \quad x \in \Omega(t) .
$$

Remark 2.1. We recall 25] that a weak solution of (1.2) is defined as satisfying the weak formulation

$$
\begin{aligned}
((f, \phi))_{G^{n}}= & -\left(\left(u, \partial_{t} \phi\right)\right)_{G^{n}}+((\nabla u, \nabla \phi))_{G^{n}} \\
& +(u, \phi)_{\Omega^{n+1}}-(u, \phi)_{\Omega^{n}}-((\beta u, \phi))_{G^{n}},
\end{aligned}
$$

for any Lipschitz continuous $\phi$ defined on $\overline{G^{n}}$ and $0 \leq n \leq N-1$. Clearly, if the solution u of (1.2) satisfies, for any $0 \leq n \leq N-1$,

$$
u \in L^{2}\left(G^{n}\right), \quad\|\nabla u\|_{G^{n}}<\infty, \quad u \in L^{2}\left(\Omega^{n}\right) \cap L^{2}\left(\Omega^{n+1}\right), \quad f \in L^{2}(G(0, T)),
$$

then it is a weak solution in the above sense. See 4, 7, for an analogous argument for the linear Schrödinger equation. Of course, $u$ may be more regular. If $f \in L^{2}(G(0, T)), u^{0} \in L^{2}(\Omega(0))$, then a weak solution to (1.2) exists, 25, 30, 33. Under additional regularity assumptions on $f$ and $u^{0}$, a strong solution $u$ exists, cf. 25, 20, 23, and in this case $u$ is continuous in $t$, and $u \in H^{1}(G(0, T))$, where

$$
\|u\|_{H^{1}(G(0, T))}=\left(\|u\|_{L^{2}(G(0, T))}^{2}+\|\nabla u\|_{L^{2}(G(0, T))}^{2}+\left\|u_{t}\right\|_{L^{2}(G(0, T))}^{2}\right)^{1 / 2} .
$$


Our a posteriori error analysis in this work, for general $\Omega(t)$, assumes that $u$ is at least continuous in time for any $t \in\left[0, t^{N}\right]$, and that $u \in H^{1}\left(G\left(0, t^{N}\right)\right)$, where $t^{N}=T$, as in 25] where the numerical scheme was proposed. Moreover, the solution $u_{h}(x, t)$ of $(1.3)$, satisfies $u_{h}^{n}(x) \simeq \lim _{\epsilon \rightarrow 0^{+}} u\left(x, t^{n}-\epsilon\right)$ and hence, $u_{h}^{N}(x) \simeq \lim _{\epsilon \rightarrow 0^{+}} u\left(x, t^{N}-\epsilon\right)$.

Remark 2.2. As we shall see later, in a case of interest, where $\Omega(t)$ is in $\mathbb{R}^{2}$, the lateral boundary $\Gamma(t)$ of $\Omega(t)$ consists of a horizontal plane and a surface $s=s(t, \theta)$, a suitable change of variables leads to a second order parabolic equation with an $s$-dependent initial condition and space-time coefficients involving s, $s_{t}$, and up to second order derivatives of $s$ in space (cf. (2.16)). The regularity of the solution of the equivalent parabolic equation depends on the regularity of the initial condition and the regularity of the coefficients. In this case we assume (2.21) for $u^{0}$, $f$ and $s$, up to $n=N$, which yields the continuity of $u$ and subsequently that $u \in H^{1}(G(0, T))$.

Remark 2.3. Any loss of continuity or of smoothness of $\Omega(t)$ and of the lateral boundary $\Gamma(t)$ for some $t$, may give rise to a non smooth or discontinuous solution $u$ at $t$. Consider, for example, problem (2.16), where such 'critical' points $t$ appear when $s$ is discontinuous (or non smooth) there, specifically when $s$ does not satisfy (2.21). These points are, nevertheless, computable since the surface s is known. Our numerical scheme is, of course, applicable up to the first critical point and the relevant error analysis is valid.

In contrast, (1.2), or (2.16), is a linear, parabolic initial and boundary value problem, and thus, any loss of regularity is induced by the initial data. So, if $t^{\prime}$ is a 'critical' point where, for example, (2.21) is not valid, we consider the time interval $\left(t, t^{\prime}\right)$ and define as initial time the time $t$. We then apply again our scheme, but now with initial condition not the exact solution $u^{N}:=\lim _{\epsilon \rightarrow 0^{+}} u(\cdot, t-\epsilon)$, as we did in (0,t) but the already computed approximation $u_{h}^{N} \simeq \lim _{\epsilon \rightarrow 0^{+}} u(\cdot, t-\epsilon)$.

The exact solution $u$ is smooth on any interval of the form $(\tau-\epsilon, \tau+\epsilon) \subset(0, t)$, for any $\tau<t$, therefore, $u^{N}$ exists and is regular, while any loss of regularity of $u(\tau)$ for $\tau>t$ is due to the low regularity of the coefficients of the pde, involving $s(\tau, \cdot)$ and its derivatives, on intervals of the form $(t-\epsilon, t+\epsilon)$ In this case, a continuous dependence of the solution $u(\cdot, \tau)$ on the exact initial condition $u^{N}$ for $\tau>t$ near $t$, is not expected. Moreover, the linear system providing the numerical solution at an interval of the form $\left(t_{\text {new }}^{0}:=t=t^{N}, t_{\text {new }}^{1}\right)$ may change significantly, mainly due to possible jumps of $s$ and its derivatives of order $\mathcal{O}(1)$ at $t^{N}$, while the contribution of the initial condition jump $u_{h}^{N}-u^{N}$ in the system is controlled by the discretization parameters. Specifically, up to time $t^{\prime}$ the schemes proposed in this work are again well posed and the a priori and a posteriori error estimates, involve an additional term from the non zero difference

$$
\varepsilon_{\text {new }}^{0}:=\varepsilon^{N}=\varepsilon\left(\cdot, t^{N}\right)=\lim _{\epsilon \rightarrow 0^{+}} u\left(\cdot, t^{N}-\epsilon\right)-u_{h}^{N}
$$

see, for example, (2.4), which is the term $\frac{1}{2}\left|\varepsilon^{N}\right|_{\Omega^{N}}^{2} \neq 0$; this term however is estimated by the a posteriori error on the interval $(0, t)$ and it is of the same order as the error in $(0, t)$, see $(2.23)$, or Theorem 3.6. Thus, our analysis is applicable under low regularity assumptions for $u$, when the problem (1.2) is posed on $S_{T}$, even discontinuity, on a finite subset of $(0, T)$, and the a posteriori estimates hold true. The case of blow-up for $s$ and its derivatives in finite time in $(0, T)$ is not covered, since we need at least, piecewise in $(0, T), L^{\infty}$ smoothness, in space and time variables, (see for example (2.21)); see also the coefficients for the more general problem 3.1 .

In what follows and for the rest of this paper, the initial condition for our numerical scheme is the exact initial condition of the continuous problem, i.e. $u_{h}^{0}=u^{0}$. However, in order to address the more general case where $u_{h}^{0} \neq u^{0}$, which, as stated in the previous remark, may give rise to a non smooth solution $u$, we retain the initial error term $\varepsilon^{0}$ in our estimates. Finally, we shall use $c$ and $C$ to denote generic constants, independent of the discretization parameters. 
Using (1.3), 1.4 , 2.1) and the continuity of $u$ in time, we see that the error $\varepsilon:=u-u_{h}$ satisfies, for any $0 \leq i \leq n-1$,

$$
\begin{aligned}
& -((\beta \varepsilon, \varepsilon))_{G^{i}}-\left(\left(\varepsilon, \partial_{t} \varepsilon\right)\right)_{G^{i}}+((\nabla \varepsilon, \nabla \varepsilon))_{G^{i}}+\left(\varepsilon^{i+1}, \varepsilon^{i+1}\right)_{\Omega^{i+1}}-\left(\varepsilon^{i}, \varepsilon^{i+0}\right)_{\Omega^{i}}= \\
& -\left(\left(\varepsilon, \partial_{t}\left(u-v_{h}\right)\right)\right)_{G^{i}}+\left(\left(\nabla \varepsilon, \nabla\left(u-v_{h}\right)\right)\right)_{G^{i}} \\
& +\left(\varepsilon^{i+1}, u^{i+1}-v_{h}^{i+1}\right)_{\Omega^{i+1}}-\left(\varepsilon^{i}, u^{i}-v_{h}^{i+0}\right)_{\Omega^{i}}-\left(\left(\beta \varepsilon, u-v_{h}\right)\right)_{G^{i}} .
\end{aligned}
$$

Let us define, as in [27, $\eta:=\varepsilon-v_{h}$ and $\eta^{n}:=\varepsilon^{n}-v_{h}^{n}$. We note that

$$
\eta^{i}+u_{h}^{i}=u^{i}-v_{h}^{i}, \quad v_{h}^{i}=u^{i}-\eta^{i}-u_{h}^{i}, \quad v_{h}^{i+0}=u^{i+0}-\eta^{i+0}-u_{h}^{i+0} .
$$

Using again the continuity of $u$ in time and (2.3) we obtain, for any $n \leq N$,

$$
\begin{aligned}
& -\sum_{i=0}^{n-1}((\beta \varepsilon, \varepsilon))_{G^{i}}+\|\nabla \varepsilon\|_{G\left(0, t^{n}\right)}^{2}+\frac{1}{2}\left|\varepsilon^{n}\right|_{\Omega^{n}}^{2}+\frac{1}{2} \sum_{i=0}^{n-1}\left|u_{h}^{i+0}-u_{h}^{i}\right|_{\Omega^{i}}^{2}= \\
& \frac{1}{2}\left|\varepsilon^{0}\right|_{\Omega^{0}}^{2}+((f, \eta))_{G\left(0, t^{n}\right)}+\sum_{i=0}^{n-1}\left(\left(u_{h}, \partial_{t} \eta\right)\right)_{G^{i}}-\sum_{i=0}^{n-1}\left(\left(\nabla u_{h}, \nabla \eta\right)\right)_{G^{i}} \\
& +\left(u_{h}^{0}, \eta^{0}\right)_{\Omega^{0}}-\left(u_{h}^{n}, \eta^{n}\right)_{\Omega^{n}}-\sum_{i=0}^{n-1}\left(u_{h}^{i}, \eta^{i}-\eta^{i+0}\right)_{\Omega^{i}}+\sum_{i=0}^{n-1}\left(\left(\beta u_{h}, \eta\right)\right)_{G^{i}},
\end{aligned}
$$

where $\varepsilon^{0}=0$ if $u_{h}^{0}=u^{0}$. Let $\mathcal{T}_{h}^{n}$ be a partition of $\overline{G^{n}}$ and

$$
V_{h}^{n}=\left\{z_{h} \in \widetilde{H}^{1}\left(G^{n}\right):\left.z_{h}\right|_{K} \in P_{\rho-1}(K), \forall K \in \mathcal{T}_{h}^{n}\right\},
$$

where $P_{\rho-1}$ is the space of polynomials of total degree at most $\rho-1$ in the time and space variables. We let $h_{n}$ denote the maximum element diameter in the partition $\mathcal{T}_{h}^{n}$ and define $h:=\max _{n} h_{n}$. Further, if $\ell$ is an interior edge of $\mathcal{T}_{h}^{n}$ we let

$$
\left[\nabla u_{h} \cdot \mathbf{n}\right]_{\ell}:=\left.\nabla u_{h} \cdot \mathbf{n}\right|_{\ell^{+}}-\left.\nabla u_{h} \cdot \mathbf{n}\right|_{\ell^{-}}
$$

denote the jump of $\nabla u_{h} \cdot \mathbf{n}$ across the edge $\ell$, where $\mathbf{n}$ is the normal direction. We also denote by $E_{K}^{n}$ the set of the edges of an element $K \in \mathcal{T}_{h}^{n}$.

Theorem 2.4. If $u$ is the solution of (1.2) and $u_{h}$ the solution of (1.3), then the error $\varepsilon=u-u_{h}$ satisfies

$$
\begin{aligned}
& -\sum_{i=0}^{n-1}((\beta \varepsilon, \varepsilon))_{G^{i}}+\|\nabla \varepsilon\|_{G\left(0, t^{n}\right)}^{2}+\frac{1}{2}\left|\varepsilon^{n}\right|_{\Omega^{n}}^{2}+\frac{1}{2} \sum_{i=0}^{n-1}\left|u_{h}^{i+0}-u_{h}^{i}\right|_{\Omega^{i}}^{2}= \\
& \quad \frac{1}{2}\left|\varepsilon^{0}\right|_{\Omega^{0}}^{2}+\sum_{i=0}^{n-1} \sum_{K \in \mathcal{T}_{h}^{i}}\left(\left(f-\partial_{t} u_{h}+\Delta u_{h}+\beta u_{h}, \eta\right)\right)_{K} \\
& \quad+\sum_{i=0}^{n-1}\left(u_{h}^{i}-u_{h}^{i+0}, \eta^{i+0}\right)_{\Omega^{i}}-\sum_{i=0}^{n-1} \sum_{K \in \mathcal{T}_{h}^{i}} \sum_{\ell \in E_{K}^{i} \text { in }} \int_{\ell} \eta\left[\nabla u_{h} \cdot \boldsymbol{n}\right]_{\ell} d s,
\end{aligned}
$$

for any $v_{h} \in V_{h}$, and for any $n \leq N$, where $\eta=\varepsilon-v_{h}$ and $E_{K, \text { in }}^{i}$ is the set of interior edges of an element $K$ of the partition $\mathcal{T}_{h}^{i}$, and $\varepsilon^{0}=0$ if $u_{h}^{0}:=u^{0}$.

Proof. Using integration by parts we have

$$
\begin{aligned}
& \left(\left(u_{h}, \partial_{t} \eta\right)\right)_{G^{i}}=-\left(\left(\partial_{t} u_{h}, \eta\right)\right)_{G^{i}}+\left(u_{h}^{i+1}, \eta^{i+1}\right)_{\Omega^{i+1}}-\left(u_{h}^{i+0}, \eta^{i+0}\right)_{\Omega^{i}} \\
& =-\left(\left(\partial_{t} u_{h}, \eta\right)\right)_{G^{i}}+\left(u_{h}^{i+1}, \eta^{i+1}\right)_{\Omega^{i+1}}-\left(u_{h}^{i}, \eta^{i+0}-\eta^{i}\right)_{\Omega^{i}}-\left(u_{h}^{i}, \eta^{i}\right)_{\Omega^{i}}+\left(u_{h}^{i}-u_{h}^{i+0}, \eta^{i+0}\right)_{\Omega^{i}},
\end{aligned}
$$




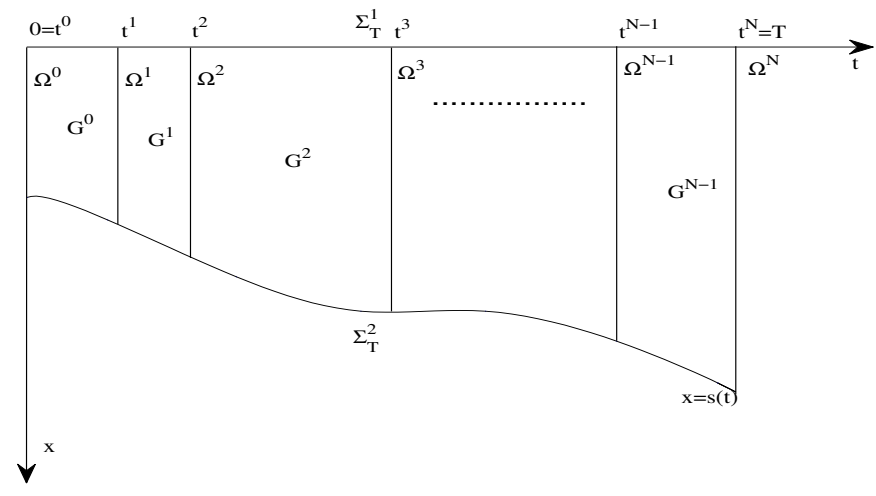

Figure 2.1. The space-time domain in the case $m=\operatorname{dim}(\Omega(t))=1, \Omega(t)=(0, s(t))$, $\Gamma(t)=\{0, s(t)\}, t \in(0, T)$ and $\Sigma_{T}=\Sigma_{T}^{1} \cup \Sigma_{T}^{2}$.

and thus

$$
\begin{aligned}
\sum_{i=0}^{n-1}\left(\left(u_{h}, \partial_{t} \eta\right)\right)_{G^{i}}= & -\sum_{i=0}^{n-1}\left(\left(\partial_{t} u_{h}, \eta\right)\right)_{G^{i}}+\left(u_{h}^{n}, \eta^{n}\right)_{\Omega^{n}}-\left(u_{h}^{0}, \eta^{0}\right)_{\Omega^{0}} \\
& +\sum_{i=0}^{n-1}\left(u_{h}^{i}, \eta^{i}-\eta^{i+0}\right)_{\Omega^{i}}+\sum_{i=0}^{n-1}\left(u_{h}^{i}-u_{h}^{i+0}, \eta^{i+0}\right)_{\Omega^{i}}
\end{aligned}
$$

In addition,

$$
\sum_{i=0}^{n-1}\left(\left(\nabla u_{h}, \nabla \eta\right)\right)_{G^{i}}=-\sum_{i=0}^{n-1} \sum_{K \in \mathcal{T}_{h}^{i}}\left(\left(\Delta u_{h}, \eta\right)\right)_{K}+\sum_{i=0}^{n-1} \sum_{K \in \mathcal{T}_{h}^{i}} \sum_{\ell \in E_{K}^{i} \text { in }} \int_{\ell} \eta\left[\nabla u_{h} \cdot \mathbf{n}\right]_{\ell} d s .
$$

Here, we used the fact that $\eta$ satisfies the Dirichlet boundary condition along the lateral boundary of $G^{i}$ and that $\eta$ is continuous in space variables Then, using (2.4), we obtain (2.5).

2.2. Upper bound. We take $\rho=2$ so that our finite element space consists of piecewise linear functions in the $t$ and $x$ variables. Let $l_{n}$ be the minimum diameter of elements in $\mathcal{T}_{h}^{n}$ and $l=\min _{n} l_{n}$. Since $\operatorname{dim}(\Omega(t))=m$ we have $\operatorname{dim} \overline{G^{n}}=m+1$ and the interior elements of the partition consist o $(m+1)$ simplices, while the boundary elements are assumed to be compatible with a possibly curved boundary, in the sense of Bernardi [11. We shall assume that there exists a positive constant $c_{0}$ such that $h / l \leq c_{0}$, meaning that the partition is regular, uniformly in $n$. To derive an upper bound for the a posteriori error we shall choose $v_{h}$ in Theorem 2.4 appropriately: For $n \geq 1$, we define $\left.v_{h}\right|_{G^{n}}$ restricted in every element $K$ of $\mathcal{T}_{h}^{n}$ as the Clément's interpolant $\pi_{h}^{n} \varepsilon$ of the error $\varepsilon$ in $P_{1}(K)$, [16, 11. We recall [16, 11, 10, that there exist positive constants, depending only on $c_{0}$, such that,

$$
\begin{aligned}
& \left\|\varepsilon-\pi_{h}^{n} \varepsilon\right\|_{K} \leq C h\|\varepsilon\|_{1, \Delta K}, \\
& \left\|\varepsilon-\pi_{h}^{n} \varepsilon\right\|_{\ell} \leq C h^{1 / 2}\|\varepsilon\|_{1, \Delta K},
\end{aligned}
$$

where $\ell$ is an edge of $K$ and $\Delta K$ denotes the set of elements having an edge or vertex common with $K$. Here, $\|\cdot\|_{\mathcal{A}},\|\cdot\|_{1, \Delta K}$ are the $L^{2}(\mathcal{A})$ and $H^{1}(\Delta K)$ norms, respectively, in $t$ and $x$ variables for $\mathcal{A}=K, \ell$. In 
particular, we note that

$$
\|\varepsilon\|_{1, \Delta K}:=\left(\|\varepsilon\|_{\Delta K}^{2}+\left\|\partial_{t} \varepsilon\right\|_{\Delta K}^{2}+\|\nabla \varepsilon\|_{\Delta K}^{2}\right)^{1 / 2}
$$

for $\nabla:=\nabla_{x}$. In the next proposition we use the estimates for the interpolant of Clément to establish an upper bound for the error.

Proposition 2.5. Let $m \geq 2$. If $u$ is the solution of (1.2), $u_{h}$ the solution of (1.3) and $\varepsilon=u-u_{h}$, then for any $0<\epsilon \leq 1$, there exist positive constants $c, c_{1}, c_{2}, c_{3}$ such that

$$
\begin{aligned}
& \sum_{i=0}^{n-1} \sum_{K \in \mathcal{T}_{h}^{i}}\|\nabla \varepsilon\|_{K}^{2}+c_{1} \sum_{i=0}^{n-1} \sum_{K \in \mathcal{T}_{h}^{i}}\|\varepsilon\|_{K}^{2}+c_{2}\left|\varepsilon^{n}\right|_{\Omega^{n}}^{2}+c_{3} \sum_{i=0}^{n-1}\left|u_{h}^{i+0}-u_{h}^{i}\right|_{\Omega^{i}}^{2} \leq \\
& \quad c\left|\varepsilon^{0}\right|_{\Omega^{0}}^{2}+c h^{2 /(1+\epsilon)} \sum_{i=0}^{n-1} \sum_{K \in \mathcal{T}_{h}^{i}}\left\|f-\partial_{t} u_{h}+\Delta u_{h}+\beta u_{h}\right\|_{L^{\vartheta}(K)} \\
& \quad+c h^{2} \sum_{i=0}^{n-1} \sum_{K \in \mathcal{T}_{h}^{i}}\left\|f-\partial_{t} u_{h}+\Delta u_{h}+\beta u_{h}\right\|_{L^{\vartheta}(K)}^{\vartheta} \\
& \quad+c h^{1 /(1+\epsilon)} \sum_{i=0}^{n-1} \sum_{K \in \mathcal{T}_{h}^{i}} \sum_{\ell \in E_{K}^{i} \text { in }}\left(\int_{\ell}\left|\left[\nabla u_{h} \cdot \boldsymbol{n}\right]_{\ell}\right|^{\vartheta} d s\right)^{1 / \vartheta} \\
& \quad+c h \sum_{i=0}^{n-1} \sum_{K \in \mathcal{T}_{h}^{i}} \sum_{\ell \in E_{K}^{i} \text { in }} \int_{\ell}\left|\left[\nabla u_{h} \cdot \boldsymbol{n}\right]_{\ell}\right|^{\vartheta} d s+c_{0} h^{1-\epsilon} \sum_{i=0}^{n-1} \sum_{K \in \mathcal{T}_{h}^{i}}\left\|\partial_{t} \varepsilon\right\|_{K}^{2},
\end{aligned}
$$

provided that $h$ is sufficiently small. Here, $\vartheta:=(1+\epsilon) / \epsilon$ and $c_{0}$ a positive constant, as small as needed. Moreover, $\varepsilon^{0}=0$, if $u_{h}^{0}:=u^{0}$.

Proof. In view of equation 2.5, we shall need estimates for the quantities $\left|\eta^{i+0}\right|_{\Omega^{i}}$, and $\|\eta\|_{\ell}$ for $\ell \in E_{K \text {,in }}^{i}$. To this end, using the second estimate of 2.6 for $\ell \in E_{K, \text { in }}^{i}$ we obtain

$$
\left(\int_{\ell}|\eta|^{2} d s\right)^{1 / 2}=\left(\int_{\ell}\left|\varepsilon-\pi_{h}^{i} \varepsilon\right|^{2} d s\right)^{1 / 2} \leq c\left(h\|\varepsilon\|_{1, \Delta K}^{2}\right)^{1 / 2}=c \sqrt{h}\|\varepsilon\|_{1, \Delta K} .
$$

Now, observe that $\Omega^{i} \subset \partial G^{i}, \partial G^{i-1}$, so that

$$
\left|\eta^{i+0}\right|_{\Omega^{i}}^{2}=\int_{\Omega^{i}}\left|\eta\left(x, t^{i+0}\right)\right|^{2} d x \leq \int_{\partial G^{i}}|\eta(x, t)|^{2} d s .
$$

By the local trace inequality (5.1) we arrive at

$$
\left|\eta^{i+0}\right|_{\Omega^{i}}^{2} \leq c \int_{\partial G^{i}}|\eta(x, t)|^{2} d s \leq c\|\eta\|_{G^{i}}\|\eta\|_{1, G^{i}}+c h^{-1}\|\eta\|_{G^{i}}^{2} .
$$

Note that the same argument gives, for $i \geq 1$,

$$
\left|\eta^{i}\right|_{\Omega^{i}}^{2} \leq \int_{\partial G^{i}}|\eta(x, t)|^{2} d s \leq c\|\eta\|_{G^{i-1}}\|\eta\|_{1, G^{i-1}}+c h^{-1}\|\eta\|_{G^{i-1}}^{2} .
$$

Assuming now that any element of $\mathcal{T}_{h}^{i}$ has a bounded number of edges, independent of $i$, we arrive at

$$
c_{1}\|a\|_{G^{i}} \leq \sum_{K \in T_{h}^{i}}\|a\|_{\Delta K} \leq c_{2}\|a\|_{G^{i}},
$$

where $c_{1}, c_{2}$ are positive constants. Using the result of [1], we also note that 2.9] gives

$$
\left|\eta^{i+0}\right|_{\Omega^{i}}^{2} \leq c h \sum_{K \in \mathcal{T}_{h}^{i}}\|\varepsilon\|_{1, \Delta K} c h^{0}\|\varepsilon\|_{1, \Delta K}+c h^{-1} h^{2} \sum_{K \in \mathcal{T}_{h}^{i}}\|\varepsilon\|_{1, \Delta K}^{2} \leq c h \sum_{K \in \mathcal{T}_{h}^{i}}\|\varepsilon\|_{1, \Delta K}^{2} .
$$


We set

$$
\mathcal{F}:=c_{0} \sum_{i=0}^{n-1} \sum_{K \in \mathcal{T}_{h}^{i}}\|\varepsilon\|_{\Delta K}^{2}+c_{1} \sum_{i=0}^{n-1} \sum_{K \in \mathcal{T}_{h}^{i}}\|\nabla \varepsilon\|_{\Delta K}^{2}+\frac{1}{2}\left|\varepsilon^{n}\right|_{\Omega^{n}}^{2}+\frac{1}{2} \sum_{i=0}^{n-1}\left|u_{h}^{i+0}-u_{h}^{i}\right|_{\Omega^{i}}^{2} .
$$

We observe that, for $0<\epsilon \leq 1,(2.6)$ and Young's inequality give

$$
\int_{\ell}|\eta|^{1+\epsilon} d s \leq \int_{\ell}\left[c+|\eta|^{2}\right] d s \leq c h+\int_{\ell}|\eta|^{2} d s \leq c h+c h\|\varepsilon\|_{1, \Delta K}^{2}
$$

which yields

$$
\left(\int_{\ell}|\eta|^{1+\epsilon} d s\right)^{1 /(1+\epsilon)} \leq c h^{1 /(1+\epsilon)}+c h^{1 /(1+\epsilon)}\|\varepsilon\|_{1, \Delta K}^{2 /(1+\epsilon)} .
$$

The same argument, applied on $K$ and using the fact that the volume of $K$ is of order $\mathcal{O}\left(h^{2}\right)$, yields

$$
\|\eta\|_{L^{1+\epsilon}(K)} \leq c h^{2 /(1+\epsilon)}+c h^{2 /(1+\epsilon)}\|\varepsilon\|_{1, \Delta K}^{2 /(1+\epsilon)}
$$

By Young's inequality we also have, for any $\gamma>0$,

$$
\begin{gathered}
\gamma h^{1 /(1+\epsilon)}\|\varepsilon\|_{1, \Delta K}^{2 /(1+\epsilon)} \leq \gamma^{(1+\epsilon) / \epsilon} h+h^{1-\epsilon}\|\varepsilon\|_{1, \Delta K}^{2}, \\
\gamma h^{2 /(1+\epsilon)}\|\varepsilon\|_{1, \Delta K}^{2 /(1+\epsilon)} \leq \gamma^{(1+\epsilon) / \epsilon} h^{2}+h^{2-2 \epsilon}\|\varepsilon\|_{1, \Delta K}^{2} .
\end{gathered}
$$

We now use 2.8, 2.10), 2.6), the fact that $\beta<0$ and 2.11, 2.12, 2.13, 2.14, to obtain, using Hölder's inequality with exponents $1+\epsilon$ and $\vartheta=(1+\epsilon) / \epsilon$, that

$$
\begin{aligned}
& \mathcal{F} \leq c_{3} \sum_{i=0}^{n-1} \sum_{K \in \mathcal{T}_{h}^{i}}\left\|f-\partial_{t} u_{h}+\Delta u_{h}+\beta u_{h}\right\|_{L^{\vartheta}(K)}\left[c h^{2 /(1+\epsilon)}+c h^{2 /(1+\epsilon)}\|\varepsilon\|_{1, \Delta K}^{2 /(1+\epsilon)}\right] \\
& +\sum_{i=0}^{n-1}\left|u_{h}^{i}-u_{h}^{i+0}\right|_{\Omega^{i}}\left|\eta^{i+0}\right|_{\Omega^{i}}+\sum_{i=0}^{n-1} \sum_{K \in \mathcal{T}_{h}^{i}} \sum_{\ell \in E_{K}^{i} \text { in }}\left(\int_{\ell}|\eta|^{(1+\epsilon)} d s\right)^{1 /(1+\epsilon)}\left(\int_{\ell}\left|\left[\nabla u_{h} \cdot \mathbf{n}\right]_{\ell}\right|^{\vartheta} d s\right)^{1 / \vartheta} \leq \\
& c h^{2 /(1+\epsilon)} \sum_{i=0}^{n-1} \sum_{K \in \mathcal{T}_{h}^{i}}\left\|f-\partial_{t} u_{h}+\Delta u_{h}+\beta u_{h}\right\|_{L^{\vartheta}(K)}+c h^{2} \sum_{i=0}^{n-1} \sum_{K \in \mathcal{T}_{h}^{i}}\left\|f-\partial_{t} u_{h}+\Delta u_{h}+\beta u_{h}\right\|_{L^{\vartheta}(K)}^{\vartheta} \\
& +c h^{2-2 \epsilon} \sum_{i=0}^{n-1} \sum_{K \in \mathcal{T}_{h}^{i}}\|\varepsilon\|_{1, \Delta K}^{2}+c_{0} \sum_{i=0}^{n-1}\left|u_{h}^{i}-u_{h}^{i+0}\right|_{\Omega^{i}}^{2}+\sum_{i=0}^{n-1}\left|\eta^{i+0}\right|_{\Omega^{i}}^{2} \\
& +\sum_{i=0}^{n-1} \sum_{K \in \mathcal{T}_{h}^{i}} \sum_{\ell \in E_{K}^{i} \text { in }}\left[c h^{1 /(1+\epsilon)}+\operatorname{ch}^{1 /(1+\epsilon)}\|\varepsilon\|_{1, \Delta K}^{2 /(1+\epsilon)}\right]\left(\int_{\ell} \|\left.\left[\nabla u_{h} \cdot \mathbf{n}\right]_{\ell}\right|^{\vartheta} d s\right)^{1 / \vartheta} \leq \\
& c h^{2 /(1+\epsilon)} \sum_{i=0}^{n-1} \sum_{K \in \mathcal{T}_{h}^{i}}\left\|f-\partial_{t} u_{h}+\Delta u_{h}+\beta u_{h}\right\|_{L^{\vartheta}(K)}+c h^{2} \sum_{i=0}^{n-1} \sum_{K \in \mathcal{T}_{h}^{i}}\left\|f-\partial_{t} u_{h}+\Delta u_{h}+\beta u_{h}\right\|_{L^{\vartheta}(K)}^{\vartheta} \\
& +c h^{2-2 \epsilon} \sum_{i=0}^{n-1} \sum_{K \in \mathcal{T}_{h}^{i}}\|\varepsilon\|_{1, \Delta K}^{2}+c_{0} \sum_{i=0}^{n-1}\left|u_{h}^{i}-u_{h}^{i+0}\right|_{\Omega^{i}}^{2}+c h \sum_{i=0}^{n-1} \sum_{K \in \mathcal{T}_{h}^{i}}\|\varepsilon\|_{1, \Delta K}^{2} \\
& +\operatorname{ch}^{1 /(1+\epsilon)} \sum_{i=0}^{n-1} \sum_{K \in \mathcal{T}_{h}^{i}} \sum_{\ell \in E_{K}^{i} \text { in }} c\left(\int_{\ell}\left|\left[\nabla u_{h} \cdot \mathbf{n}\right]_{\ell}\right|^{\vartheta} d s\right)^{1 / \vartheta} \\
& +c h^{1 /(1+\epsilon)} \sum_{i=0}^{n-1} \sum_{K \in \mathcal{T}_{h}^{i}} \sum_{\ell \in E_{K}^{i} \text { in }} c \int_{\ell}\left\|\left.\left[\nabla u_{h} \cdot \mathbf{n}\right]_{\ell}\right|^{\vartheta} d s+c_{0} h^{1-\epsilon} \sum_{i=0}^{n-1} \sum_{K \in \mathcal{T}_{h}^{i}}\right\| \varepsilon \|_{1, \Delta K}^{2} .
\end{aligned}
$$


Since

we finally arrive at

$$
\|\varepsilon\|_{1, \Delta K} \leq c\|\varepsilon\|_{\Delta K}+c\|\nabla \varepsilon\|_{\Delta K}+c\left\|\varepsilon_{t}\right\|_{\Delta K},
$$

$$
\begin{aligned}
& c \sum_{i=0}^{n-1} \sum_{K \in \mathcal{T}_{h}^{i}}\|\varepsilon\|_{\Delta K}^{2}+c_{1} \sum_{i=0}^{n-1} \sum_{K \in \mathcal{T}_{h}^{i}}\|\nabla \varepsilon\|_{\Delta K}^{2}+c_{2}\left|\varepsilon^{n}\right|_{\Omega^{n}}^{2}+c_{3} \sum_{i=0}^{n-1}\left|u_{h}^{i+0}-u_{h}^{i}\right|_{\Omega^{i}}^{2} \leq \\
& c h^{2 /(1+\epsilon)} \sum_{i=0}^{n-1} \sum_{K \in \mathcal{T}_{h}^{i}}\left\|f-\partial_{t} u_{h}+\Delta u_{h}+\beta u_{h}\right\|_{L^{\vartheta}(K)}+c h^{2} \sum_{i=0}^{n-1} \sum_{K \in \mathcal{T}_{h}^{i}}\left\|f-\partial_{t} u_{h}+\Delta u_{h}+\beta u_{h}\right\|_{L^{\vartheta}(K)}^{\vartheta} \\
& +c_{0} h^{1-\epsilon} \sum_{i=0}^{n-1} \sum_{K \in \mathcal{T}_{h}^{i}}\left\|\varepsilon_{t}\right\|_{\Delta K}^{2}+c h \sum_{i=0}^{n-1} \sum_{K \in \mathcal{T}_{h}^{i}} \sum_{\ell \in E_{K}^{i} \text { in }} \int_{\ell}\left|\left[\nabla u_{h} \cdot \mathbf{n}\right]_{\ell}\right|^{\vartheta} d s \\
& +c h^{1 /(1+\epsilon)} \sum_{i=0}^{n-1} \sum_{K \in \mathcal{T}_{h}^{i}} \sum_{\ell \in E_{K}^{i} \text { in }}\left(\int_{\ell}\left|\left[\nabla u_{h} \cdot \mathbf{n}\right]_{\ell}\right|^{\vartheta} d s\right)^{1 / \vartheta},
\end{aligned}
$$

for a positive constant $c_{0}$, as small as desired and $h$ sufficiently small. The result now follows from 2.5).

We assume now that the non-cylindrical domain $S_{T}$ is two dimensional in the spatial variables and $\Omega(t)$ is of the form

$$
\Omega(t):=\left\{(z, \theta) \in R^{2}: \quad z \in(0, s(t, \theta)), \quad \theta \in\left(\theta_{1}, \theta_{2}\right)\right\}, \quad \text { for } t \in[0, T],
$$

where $s$ is a smooth, positive function. Specific smoothness assumptions are given later. In this case, for $g$ a sufficiently smooth function it follows that

$$
\int_{0}^{t} \int_{\Omega(t)} g d x d t=\int_{0}^{t} \int_{\theta_{1}}^{\theta_{2}} \int_{0}^{s(t, \theta)} g d z d \theta d t
$$

For a given $t$, the boundary $\Gamma(t)$ of $\Omega(t)$ consists of the surfaces $z=0, z=s(t, \theta), \theta=\theta_{1}, \theta_{2}$. We apply the transformation [4, 1, 7, 5]

$$
y:=\frac{z}{s}, \quad \hat{u}(y, \theta, t):=u(z, \theta, t) .
$$

This change of variables gives $y \in(0,1), \Omega(t) \hookrightarrow \hat{\Omega}=: \hat{\Omega}^{i}$, for any $i$ and $t \in(0, T)$, and $S_{T} \hookrightarrow \hat{S}_{T}, G^{i} \hookrightarrow \hat{G}^{i}$, where

$$
\begin{aligned}
& \hat{\Omega}=(0,1) \times\left(\theta_{1}, \theta_{2}\right) \quad(t \text { - independent }), \\
& \hat{S}_{T}=(0, T) \times(0,1) \times\left(\theta_{1}, \theta_{2}\right) \quad(\text { cylindrical }), \\
& \hat{G}^{i}=\left(t^{i}, t^{i+1}\right) \times \hat{\Omega} .
\end{aligned}
$$

The initial and boundary value problem 1.2 now becomes

$$
\begin{aligned}
& \partial_{t} \hat{u}=A \hat{u}_{y y}+B \hat{u}_{y \theta}+C \hat{u}_{\theta \theta}+D \hat{u}_{y}+\beta_{1} \hat{u}+\hat{f} \text { in } \hat{S}_{T}, \\
& \hat{u}=0 \text { at } y=0,1, \\
& \hat{u}=0 \text { at } \theta=\theta_{1}, \theta_{2}, \\
& \hat{u}(y, \theta, 0)=u^{0}(y s(0, \theta), \theta),
\end{aligned}
$$

where $\hat{u}(y, \theta, t):=u(z, \theta, t), A=\frac{1}{s^{2}}+\frac{y^{2} s_{\theta}^{2}}{s^{2}}, B=-2 y \frac{s_{\theta}}{s}, C=1, D=\frac{y s_{t}}{s}-\frac{y}{s^{2}}\left(s_{\theta \theta} s-2 s_{\theta}^{2}\right), \beta_{1}=\beta$ and $\hat{f}(y, \theta, t)=f(z, \theta, t)$. For the purpose of the analysis below we assume that

$$
s, s_{t}, s_{\theta}, s_{\theta \theta} \in L^{\infty}\left((0, T) \times\left(\theta_{1}, \theta_{2}\right)\right),
$$

which implies that the space-time coefficients of problem (2.16) satisfy $A, B, D \in L^{\infty}\left(\hat{S}_{T}\right)$. Also, $C, \beta_{1}$ are constants and thus in $L^{\infty}\left(\hat{S}_{T}\right)$. The next lemma presents an estimate for $\left\|\partial_{t} \hat{u}\right\|$. 
Lemma 2.6. Let $\hat{u}$ be the solution of (2.16), and assume that 2.17) holds. Then, for any $t \leq T$, it holds

$$
\int_{0}^{t}\|\hat{u}\|_{H^{2}(\hat{\Omega})}^{2}+\int_{0}^{t}\left\|\hat{u}_{t}\right\|^{2} d t \leq c\|\hat{u}(0)\|_{H^{1}(\hat{\Omega})}^{2}+c \int_{0}^{t}\|\hat{f}\|^{2} d t
$$

provided that $u^{0} \in H^{1}(\Omega(0))$ and $f \in L^{2}(G(0, T))$.

Proof. The regularity of $s, s_{\theta}$ yields $\hat{u}^{0} \in H^{2 m+1}(\hat{\Omega})$ for $m=0$, provided that $u^{0} \in H^{1}(\Omega(0))$. If $\hat{f} \in$ $L^{2}\left(0, T ; L^{2}(\hat{\Omega})\right)$, or, equivalently, since $s \in L^{\infty}\left((0, T) \times\left(\theta_{1}, \theta_{2}\right)\right)$, that $f \in L^{2}(G(0, T))$, then relation (55), p. 365 of Evans [19], yields the result.

Since

$$
\partial_{t} u=\partial_{t} \hat{u}-\frac{y}{s} s_{t} \hat{u}_{y}, \quad \hat{u}_{y}=s u_{z}, \quad \hat{u}_{\theta}=y s_{\theta} u_{z}+u_{\theta},
$$

we readily obtain from 2.18 the proof of the next proposition.

Proposition 2.7. If $u$ is the solution of (1.2) then, under the assumptions of Lemma 2.6,

$$
\sum_{i=0}^{n-1} \sum_{K \in \mathcal{T}_{h}^{i}}\left\|\partial_{t} u\right\|_{K}^{2} \leq c\left|u^{0}\right|_{\Omega^{0}}^{2}+c\left|\nabla u^{0}\right|_{\Omega^{0}}^{2}+c \sum_{i=0}^{n-1} \sum_{K \in \mathcal{T}_{h}^{i}}\|f\|_{K}^{2},
$$

for any $1 \leq n \leq N$.

Remark 2.8. We note that the solution $u$ has been assumed to be continuous in time in $(0, T)$. For this, it is sufficient that $u$ belongs to $H^{2}\left(S_{T}\right)$, since $u=u(z, \theta, t)$ and $(z, \theta) \in \mathbb{R}^{2}, t \in \mathbb{R}$. For the former, it is sufficient that

$$
\int_{0}^{T}\left[\left\|\hat{u}_{t t}\right\|_{L^{2}(\hat{\Omega})}^{2}+\left\|\hat{u}_{t}\right\|_{H^{1}(\hat{\Omega})}^{2}+\|\hat{u}\|_{H^{2}(\hat{\Omega})}^{2}\right] d t<\infty .
$$

Observe that $\int_{0}^{T}\|\hat{u}\|_{H^{2}(\hat{\Omega})}^{2} d t<\infty$, under the regularity assumptions on $u^{0}, f, s$ in the proof of Lemma 2.6 The term $\left\|\hat{u}_{t}\right\|_{H^{1}(\hat{\Omega})}^{2}$ may be estimated by differentiating 2.16 in space, while $\left\|\hat{u}_{t t}\right\|_{L^{2}(\hat{\Omega})}^{2}$ may be estimated by differentiating 2.16) in time. Thus, $\int_{0}^{T}\left\|\hat{u}_{t}\right\|_{H^{1}(\hat{\Omega})}^{2}$ is bounded if $\hat{u} \in L^{2}\left(0, T ; H^{3}(\hat{\Omega})\right)$, while $\left\|\hat{u}_{t t}\right\|_{L^{2}(\hat{\Omega})}^{2}$ is bounded if $\hat{u}^{0} \in H^{3}(\hat{\Omega})$ and $\hat{f}_{t} \in L^{2}\left(0, T ; L^{2}(\hat{\Omega})\right)$. The regularity of $s, s_{\theta}$, se yields $\hat{u}^{0} \in H^{2}(\hat{\Omega})$, if $u^{0} \in H^{2}(\Omega(0))$. If we assume additionally, that $s_{t t}, s_{t \theta}, s_{t \theta \theta}, s_{\theta \theta \theta} \in L^{\infty}\left((0, T) \times\left(\theta_{1}, \theta_{2}\right)\right)$, and that $\hat{f} \in$ $L^{2}\left(0, T ; H^{1}(\hat{\Omega})\right), \quad \hat{f}_{t} \in L^{2}\left(0, T ; L^{2}(\hat{\Omega})\right)$, or, in the initial coordinates that $f, f_{t}, f_{z}, f_{\theta} \in L^{2}(G(0, T))$ then, we have $\hat{u} \in L^{2}\left(0, T ; H^{3}(\hat{\Omega})\right)$.

In view of the remarks above, we conclude that if

$$
u^{0} \in H^{3}(\Omega(0)), \quad s, s_{t}, s_{t t}, s_{t \theta}, s_{\theta}, s_{\theta \theta}, s_{t \theta \theta}, s_{\theta \theta \theta} \in L^{\infty}\left(\hat{S}_{T}\right), \quad f, f_{t}, f_{z}, f_{\theta} \in L^{2}(G(0, T)),
$$

then $u$ is continuous, and obviously, also (2.19) is valid, i.e., $u_{t} \in L^{2}(G(0, T))$ and moreover, by (2.18), $u \in H^{1}(G(0, T))$. We write $u=u-u_{h}+u_{h}=\varepsilon+u_{h}, \partial_{t} \varepsilon=\partial_{t} u-\partial_{t} u_{h}$. Using 2.19, 2.20) and this splitting we obtain the following estimate for $\varepsilon_{t}$, where in place of $T$ we may take any nodal point $t^{n} \leq T$.

Theorem 2.9. Let $u$ be the solution of (1.2) and $1 \leq n \leq N$. If

$$
u^{0} \in H^{3}(\Omega(0)), \quad s, s_{t t}, s_{t}, s_{t \theta}, s_{\theta}, s_{\theta \theta}, s_{t \theta \theta}, s_{\theta \theta \theta} \in L^{\infty}\left(\hat{S}_{T}\right), \quad f, f_{t}, f_{z}, f_{\theta} \in L^{2}\left(G\left(0, t^{n}\right)\right),
$$

then there exists a positive constant $c_{e}$ such that

$$
\sum_{i=0}^{n-1} \sum_{K \in \mathcal{T}_{h}^{i}}\left\|\varepsilon_{t}\right\|_{K}^{2} \leq c_{e}\left|u^{0}\right|_{\Omega^{0}}^{2}+c_{e}\left|\nabla u^{0}\right|_{\Omega^{0}}^{2}+c_{e} \sum_{i=0}^{n-1} \sum_{K \in \mathcal{T}_{h}^{i}}\left(\left\|\partial_{t} u_{h}\right\|_{K}^{2}+\|f\|_{K}^{2}\right),
$$

and the solution $u$ of the initial problem is continuous.

Applying (2.22) in (2.7), we have the following a posteriori upper bound. 
Theorem 2.10. Let $m=2$ and $\Omega(t)$ be given by 2.15). Let $u$ be the solution of (1.2) and $1 \leq n \leq N$. If 2.20 holds and $f \in L^{\vartheta}\left(G\left(0, t^{n}\right)\right)$ then, for any $0<\epsilon \leq 1$, and with $\vartheta:=(1+\epsilon) / \epsilon$, there exist positive constants $c_{1}, c_{2}, c_{3}, c$ such that

$$
\begin{aligned}
& \sum_{i=0}^{n-1} \sum_{K \in \mathcal{T}_{h}^{i}}\|\nabla \varepsilon\|_{K}^{2}+c_{1} \sum_{i=0}^{n-1} \sum_{K \in \mathcal{T}_{h}^{i}}\|\varepsilon\|_{K}^{2}+c_{2}\left|\varepsilon^{n}\right|_{\Omega^{n}}^{2}+c_{3} \sum_{i=0}^{n-1}\left|u_{h}^{i+0}-u_{h}^{i}\right|_{\Omega^{i}}^{2} \leq \\
& c\left|\varepsilon^{0}\right|_{\Omega^{0}}^{2}+c h^{2 /(1+\epsilon)} \sum_{i=0}^{n-1} \sum_{K \in \mathcal{T}_{h}^{i}}\left\|f-\partial_{t} u_{h}+\Delta u_{h}+\beta u_{h}\right\|_{L^{\vartheta}(K)} \\
& +c h^{2} \sum_{i=0}^{n-1} \sum_{K \in \mathcal{T}_{h}^{i}}\left\|f-\partial_{t} u_{h}+\Delta u_{h}+\beta u_{h}\right\|_{L^{\vartheta}(K)}^{\vartheta} \\
& +c h^{1 /(1+\epsilon)} \sum_{i=0}^{n-1} \sum_{K \in \mathcal{T}_{h}^{i}} \sum_{\ell \in E_{K}^{i} \text { in }}\left(\int_{\ell}\left|\left[\nabla u_{h} \cdot \boldsymbol{n}\right]_{\ell}\right|^{\vartheta} d s\right)^{1 / \vartheta} \\
& +c h \sum_{i=0}^{n-1} \sum_{K \in \mathcal{T}_{h}^{i}} \sum_{\ell \in E_{K}^{i} \text { in }} \int_{\ell}\left|\left[\nabla u_{h} \cdot \boldsymbol{n}\right]_{\ell}\right|^{\vartheta} d s \\
& +c h^{1-\epsilon}\left[\left|u^{0}\right|_{\Omega^{0}}^{2}+\left|\nabla u^{0}\right|_{\Omega^{0}}^{2}+\sum_{i=0}^{n-1} \sum_{K \in \mathcal{T}_{h}^{i}}\left(\left\|\partial_{t} u_{h}\right\|_{K}^{2}+\|f\|_{K}^{2}\right)\right],
\end{aligned}
$$

for h sufficiently small.

Remark 2.11. Obviously, in view of the terms involving $h^{1-\epsilon}$ and $h^{1 /(1+\epsilon)}$, the upper bound estimate (2.23) is suboptimal, viewed as an $L^{2}$ estimate. Moreover, 2.23 may not be seen as an $H^{1}$ estimate in the noncylindrical domain, since the time derivative of the error (of the relevant $H^{1}$ norm) does not appear in the left-hand side. This is due to the fact that the space-time discretization is applied in the initial non-cylindrical domain, and therefore, the term $\varepsilon_{t}$ reduces the order of convergence. As we shall demonstrate in the sequel, by transforming the initial problem into an equivalent one posed on a cylindrical domain, the a posteriori error analysis for the resulting scheme does indeed give optimal results in $H^{1}$ norm, see Theorem 3.6 and the remarks following that theorem.

\section{A posteriori ERROR ANALYSIS FOR THE EQUiVALENT PROBLEM}

3.1. Change of variables - The general parabolic problem. As we showed in the analysis in Section 2, the initial problem (1.2) for a case of interest when $m=2$, is transformed to an equivalent problem, cf. (2.16), posed on a cylindrical domain. We shall consider the more general parabolic problem

$$
\begin{aligned}
& \partial_{t} \hat{u}=A \hat{u}_{y y}+B \hat{u}_{y \theta}+C \hat{u}_{\theta \theta}+D \hat{u}_{y}+E \hat{u}_{\theta}+\beta_{1} \hat{u}+\hat{f} \text { in } \hat{S}_{T}, \\
& \hat{u}=0 \text { at } y=0,1, \\
& \hat{u}=0 \text { at } \theta=\theta_{1}, \theta_{2}, \\
& \hat{u}(y, \theta, 0)=\hat{u}^{0}(y, \theta),
\end{aligned}
$$

with $A, B, C, D, E, \beta_{1}$ functions of $(y, \theta, t)$, and $A, C$ positive while $B^{2}<4 A C$. Note that 2.16) is a special case of this general parabolic problem, with $E=0$. Also, we shall assume that $\beta_{1}$ is a negative function whose absolute value is controlled by the transformation $\hat{u} \rightarrow e^{c t} \hat{u}$, for a suitable constant $c$. In the sequel we shall assume that the coefficients $A, B, C, D, E, \beta_{1}$, and $\hat{u}^{0}, \hat{f}$, satisfy, for $1 \leq n \leq N$,

$$
\begin{aligned}
& \hat{u}^{0} \in H^{3}(\hat{\Omega}), \hat{f}, \hat{f}_{t}, \hat{f}_{y}, \hat{f}_{\theta} \in L^{2}\left(\hat{S}_{t^{n}}\right), \\
& \partial_{t}^{k} \partial_{x}^{\mu} \mathcal{L} \in L^{\infty}\left(\hat{S}_{t^{n}}\right) \text { for } \mathcal{L}:=A, B, C, D, E, \beta_{1}, \quad x=y, \theta, \text { and } k, \mu=0,1,
\end{aligned}
$$


A POSTERIORI ANALYSIS FOR SPACE-TIME, DISCONTINUOUS IN TIME GALERKIN APPROXIMATIONS

in $\hat{S}_{t^{n}}=\left(0, t^{n}\right) \times(0,1) \times\left(\theta_{1}, \theta_{2}\right)$. These assumptions yield that $u$ is continuous in time in $\left[0, t^{n}\right]$, and $u_{t} \in L^{2}\left(\hat{S}_{t^{n}}\right), u \in H^{1}\left(\hat{S}_{t^{n}}\right)$, as in Remark 2.8. For the problem 3.1 we define an analogous to 1.3 . space-time, discontinuous in time scheme, and seek $u_{h} \in V_{h}$ satisfying

$$
\begin{aligned}
& \hat{B}_{n}\left(u_{h}, v_{h}\right)=\left(\left(\hat{f}, v_{h}\right)\right)_{\hat{G}^{n}}, \quad \forall v_{h} \in V_{h}^{n}, \quad n=0, \ldots, N-1, \\
& u_{h}^{0}=\hat{u}^{0},
\end{aligned}
$$

where, now, the bilinear form $\hat{B}_{n}\left(u_{h}, v_{h}\right)$ is defined as

$$
\begin{aligned}
\hat{B}_{n}\left(u_{h}, v_{h}\right):= & -\left(\left(u_{h}, \partial_{t} v_{h}\right)\right)_{\hat{G}^{n}}-\int_{t^{n}}^{t^{n+1}} \mathcal{B}\left(u_{h}, v_{h}\right) d t \\
& +\left(u_{h}^{n+1}, v_{h}^{n+1}\right)_{\hat{\Omega}}-\left(u_{h}^{n}, v_{h}^{n+0}\right)_{\hat{\Omega}}-\left(\left(\widetilde{D} u_{h y}+\widetilde{E} u_{h \theta}+\beta_{1} u_{h}, v_{h}\right)\right)_{\hat{G}^{n}} .
\end{aligned}
$$

Here, $\widetilde{D}=D-A_{y}-\frac{B_{\theta}}{2}$ and $\widetilde{E}=E-C_{\theta}-\frac{B_{y}}{2}$, while $\mathcal{B}: H^{1}(\hat{\Omega}) \times H^{1}(\hat{\Omega}) \rightarrow R$ is a bilinear form given by

$$
\mathcal{B}(v, w):=-\left(A v_{y}, w_{y}\right)_{\hat{\Omega}}-\left(C v_{\theta}, w_{\theta}\right)_{\hat{\Omega}}-\frac{1}{2}\left\{\left(B v_{y}, w_{\theta}\right)_{\hat{\Omega}}+\left(B v_{\theta}, w_{y}\right)_{\hat{\Omega}}\right\}
$$

We first observe that, since $A, C>0$ and $B^{2}<4 A C$, there exists a positive constant $c$ such that

$$
-\mathcal{B}(u, u) \geq c|\nabla u|_{\hat{\Omega}}^{2} .
$$

The proof of the following existence theorem is now immediate.

Theorem 3.1. The problem (3.3) admits a unique solution $u_{h} \in V_{h}$.

Proof. We set $v_{h}=u_{h}$ in 3.3 so that

$$
-\int_{t^{n}}^{t^{n+1}} \mathcal{B}\left(u_{h}, u_{h}\right) d t-\left(\left(\widetilde{D} u_{h y}+\widetilde{E} u_{h \theta}+\beta_{1} u_{h}, u_{h}\right)\right)_{\hat{G}^{n}}+\frac{1}{2}\left|u_{h}^{n+1}\right|_{\hat{\Omega}}^{2} \leq \frac{1}{2}\left|u_{h}^{n}\right|_{\hat{\Omega}}^{2}+\left|\left(\left(\hat{f}, u_{h}\right)\right)_{\hat{G}^{n}}\right| .
$$

Then, 3.5 gives by summation

$$
\left\|u_{h}\right\|_{\hat{G}\left(0, t^{n}\right)}^{2}+\left\|\nabla u_{h}\right\|_{\hat{G}\left(0, t^{n}\right)}^{2} \leq c\left|\hat{u}^{0}\right|_{\hat{\Omega}}^{2}+c\|\hat{f}\|_{\hat{G}\left(0, t^{n}\right)}^{2},
$$

for $\beta_{1}$ sufficiently negative. This establishes the existence of a unique solution of problem (3.3).

Let $u_{h}$ be the unique solution of (3.3) in $V_{h}$ and $\varepsilon:=\hat{u}-u_{h}$, where $\hat{u}$ is the solution of the continuous problem (3.1). Also, let $\eta:=\varepsilon-v_{h}$, for $v_{h}$ an arbitrary element of $V_{h}$. By computations entirely analogous to those leading to 2.4 we obtain

$$
\begin{aligned}
& -\sum_{i=0}^{n-1}\left(\left(\widetilde{D} \varepsilon_{y}+\widetilde{E} \varepsilon_{\theta}+\beta_{1} \varepsilon, \varepsilon\right)\right)_{\hat{G}^{i}}-\int_{0}^{t^{n}} \mathcal{B}(\varepsilon, \varepsilon) d t+\frac{1}{2}\left|\varepsilon^{n}\right|_{\hat{\Omega}}^{2}+\frac{1}{2} \sum_{i=0}^{n-1}\left|u_{h}^{i+0}-u_{h}^{i}\right|_{\hat{\Omega}}^{2}= \\
& \frac{1}{2}\left|\varepsilon^{0}\right|_{\hat{\Omega}}^{2}+((\hat{f}, \eta))_{\hat{G}\left(0, t^{n}\right)}+\sum_{i=0}^{n-1}\left(\left(u_{h}, \partial_{t} \eta\right)\right)_{\hat{G}^{i}}+\sum_{i=0}^{n-1} \int_{t^{i}}^{t^{i+1}} \mathcal{B}\left(u_{h}, \eta\right) d t+\left(u_{h}^{0}, \eta^{0}\right)_{\hat{\Omega}}-\left(u_{h}^{n}, \eta^{n}\right)_{\hat{\Omega}} \\
& -\sum_{i=0}^{n-1}\left(u_{h}^{i}, \eta^{i}-\eta^{i+0}\right)_{\hat{\Omega}}+\sum_{i=0}^{n-1}\left(\left(\widetilde{D} u_{h y}+\widetilde{E} u_{h \theta}+\beta_{1} u_{h}, \eta\right)\right)_{\hat{G}^{i}} .
\end{aligned}
$$

Given the partition $0=t^{0}<t^{1} \cdots<t^{N}=T$ of $[0, T]$, we let $I_{n}=\left(t^{n}, t^{n+1}\right], \quad k_{n}=t^{n+1}-t^{n}$ and $k_{n}=\max _{0 \leq i \leq n-1} k_{i}$. Let us consider a triangulation $T_{h}^{n}$ of $\overline{\hat{\Omega}}$ and a finite element space $S_{h}^{n}$ in each interval $I_{n}$ consisting of functions in $H_{0}^{1}(\hat{\Omega})$ which reduce to polynomials of degree $r-1$ on each triangle. Here, for any $n, h_{n}$ is the maximum diameter of elements of the triangulation $T_{h}^{n}$. As before, we let $\boldsymbol{h}_{n}=\max _{0 \leq i \leq n-1} h_{i}$. Now let $\mathcal{V}_{h k}=\mathcal{V}_{h k}(q), q \geq 2$, denote the space of piecewise polynomial functions $\phi:(0, T] \times \hat{\Omega} \rightarrow R$ of the form: $\left.\phi\right|_{I_{n} \times \hat{\Omega}}=\sum_{j=0}^{q-1} t^{j} \chi_{j}(y), \chi_{j} \in S_{h}^{n}$. It follows that the functions of $\mathcal{V}_{h k}$ are, for each $t \in I_{n}$ elements of 
$S_{h}^{n}$ and for each $y \in \hat{\Omega}$ piecewise polynomial functions of degree $q-1$, with possible discontinuities at the temporal nodes $t^{n}, n=0, \ldots, N-1$. We also define $\mathcal{V}_{h k}^{n}=\left\{\left.\phi\right|_{I_{n} \times \hat{\Omega}}: \phi \in \mathcal{V}_{h k}\right\}$.

3.2. Upper bound. Let us define the symmetric matrix

$$
\mathcal{M}:=\left(\begin{array}{cc}
A & \frac{B}{2} \\
\frac{B}{2} & C
\end{array}\right) .
$$

If $\ell$ is an interior edge of $T_{h}^{n}$, let $\left[\mathcal{M} \nabla u_{h} \cdot \mathbf{n}\right]_{\ell}$ denote the jump of $\mathcal{M} \nabla u_{h} \cdot \mathbf{n}$ across the edge $\ell$, where $\mathbf{n}$ is the normal direction, i.e.

$$
\left[\mathcal{M} \nabla u_{h} \cdot \mathbf{n}\right]_{\ell}:=\left.\mathcal{M} \nabla u_{h} \cdot \mathbf{n}\right|_{\ell^{+}}-\left.\mathcal{M} \nabla u_{h} \cdot \mathbf{n}\right|_{\ell^{-}} .
$$

Moreover, we denote by $E_{K}^{n}$ the set of the edges of an element $K \in T_{h}^{n}$.

Theorem 3.2. For the error $\varepsilon=\hat{u}-u_{h}$ it holds

$$
\begin{aligned}
& -\sum_{i=0}^{n-1}\left(\left(\widetilde{D} \varepsilon_{y}+\widetilde{E} \varepsilon_{\theta}+\beta_{1} \varepsilon, \varepsilon\right)\right)_{\hat{G}^{i}}-\int_{0}^{t^{n}} \mathcal{B}(\varepsilon, \varepsilon) d t+\left.\left.\frac{1}{2}\right|^{n}\right|_{\hat{\Omega}} ^{2}+\frac{1}{2} \sum_{i=0}^{n-1}\left|u_{h}^{i+0}-u_{h}^{i}\right|_{\hat{\Omega}}^{2}= \\
& \frac{1}{2}\left|\varepsilon^{0}\right|_{\hat{\Omega}}^{2}+\sum_{i=0}^{n-1} \int_{I_{i}} \sum_{K \in T_{h}^{i}}\left(\hat{f}-\partial_{t} u_{h}+A u_{h y y}+B u_{h y \theta}+C u_{h \theta \theta}+D u_{h y}+E u_{h \theta}+\beta_{1} u_{h}, \eta\right)_{K} d t \\
& -\sum_{i=0}^{n-1} \int_{I_{i}} \sum_{K \in T_{h}^{i}} \sum_{\ell \in E_{K \text { in }}^{i}} \int_{\ell} \eta\left[\mathcal{M} \nabla u_{h} \cdot \boldsymbol{n}\right]_{\ell} d s d t+\sum_{i=0}^{n-1}\left(u_{h}^{i}-u_{h}^{i+0}, \eta^{i+0}\right)_{\hat{\Omega}},
\end{aligned}
$$

where $(\cdot, \cdot)_{K}$ is the usual inner product in $L^{2}(K)$.

For the purposes of establishing an error bound, we shall take $r=2$, so that $S_{h}^{n}$ consists of piecewise linear functions in the $y, \theta$ variables. We also assume that $T_{h}^{n}$ is regular, uniformly on any $n$. For a fixed $n$ and $t \in I_{n}$, we denote by $\pi_{h}^{n} \varepsilon(t)$ the Clément interpolant of the error $\varepsilon(t)$ in $P_{1}(K)$, where $K$ is an element of $T_{h}^{n}$. Since $r=2$, we have $\pi_{h}^{n} \varepsilon(t) \in S_{h}^{n}$. In fact, if $B_{n}=\left\{b_{i}\right\}_{i=1}^{\operatorname{dim}_{h}^{n}}$ is a basis of $S_{h}^{n}$, for any $y \in \hat{\Omega}$,

$$
\pi_{h}^{n} \varepsilon(y, \theta, t)=\sum_{i=1}^{\operatorname{dim} S_{h}^{n}} c_{i}(t) b_{i}(y, \theta),
$$

where $c_{i}(t) \in \mathbb{R}$. In order to obtain an element of $\mathcal{V}_{h k}$ we define $\left.v_{h}\right|_{\hat{G}^{n}}$ as the $L^{2}\left(I_{n}\right)$-projection of $\pi_{h}^{n} \varepsilon$ in $P_{q-1}\left(I_{n}\right)$, the polynomials of order at most $q-1$ in $t \in I_{n}$. We shall also make use of the $L^{2}$ projection operator $L^{n}: L^{2}\left(I_{n}\right) \rightarrow P_{q-1}\left(I_{n}\right)$ defined by

$$
\int_{I_{n}} t^{j} L^{n}(g(t)) d t=\int_{I_{n}} t^{j} g(t) d t, \quad \forall g \in L^{2}\left(I_{n}\right), \quad j=0, \ldots, q-1 .
$$

Let $f^{(q)}$ denote the $q$-time derivative of $f$. If $f(\cdot, t) \in C^{q}\left(\bar{I}_{n}\right)$ then, cf. [4,

$$
\int_{I_{n}}\left|L^{n} f-f\right|^{2} d t \leq c k_{n}^{2 q+1}\left(\max _{t \in \bar{I}_{n}}\left|f^{(q)}(\cdot, t)\right|\right)^{2}
$$

We can now define $v_{h} \in \mathcal{V}_{h k}$ by

$$
v_{h}{\mid \mathcal{V}_{h k}^{n}}^{n}=L^{n} \circ \pi_{h}^{n} \varepsilon \text { in } I_{n} \times \hat{\Omega}, \quad n=0, \ldots, N-1 .
$$

Obviously, it holds that

$$
L^{n} \circ \pi_{h}^{n} \varepsilon(y, \theta, t)=\sum_{i=1}^{\operatorname{dim} S_{h}^{n}} L^{n}\left(c_{i}(t)\right) b_{i}(y, \theta) .
$$


As before, we note that there exists a positive constant $C$ such that

$$
\begin{gathered}
\left\|\varepsilon-\pi_{h}^{n} \varepsilon\right\|_{L^{2}(K)} \leq C \boldsymbol{h}_{n}\|\varepsilon\|_{H^{1}(\Delta K)}, \\
\left\|\varepsilon-\pi_{h}^{n} \varepsilon\right\|_{L^{2}(\ell)} \leq C \boldsymbol{h}_{n}^{1 / 2}\|\varepsilon\|_{H^{1}(\Delta K)} .
\end{gathered}
$$

We define the $L^{2}\left(0, s ; H^{k}(\hat{\Omega})\right)$ norm as

$$
\|g\|_{L^{2}\left(0, s ; H^{k}(\hat{\Omega})\right)}:=\left(\int_{0}^{s}\|g(\cdot, t)\|_{H^{k}(\hat{\Omega})}^{2} d t\right)^{1 / 2}, \quad k=0,1, \ldots
$$

where $H^{0}(\hat{\Omega}):=L^{2}(\hat{\Omega})$ and $H^{k}(\hat{\Omega})$ is the usual Sobolev space.

Remark 3.3. Considering the solution $\hat{u}$ of the problem (3.1), if (3.2) holds also for $k, \mu=0, \ldots, m+1$, for some $m \geq 0$, then, for $1 \leq n \leq N$, and any $0<s \leq t^{n}$, we have

$$
\sum_{k=0}^{m+1}\left\|\hat{u}^{(k)}\right\|_{L^{2}\left(0, s ; H^{2 m+2-2 k}(\hat{\Omega})\right)} \leq c\left(\sum_{k=0}^{m}\left\|\hat{f}^{(k)}\right\|_{L^{2}\left(0, s ; H^{2 m-2 k}(\hat{\Omega})\right)}+\left\|\hat{u}^{0}\right\|_{H^{2 m+1}(\hat{\Omega})}\right),
$$

if $\hat{u}^{0} \in H^{2 m+1}(\hat{\Omega})$, and $\hat{f}^{(k)} \in L^{2}\left(0, s ; H^{2 m-2 k}(\hat{\Omega})\right)$ for any $k=0, \ldots, m+1$, where we have set $\hat{u}^{(k)}:=\partial_{t}^{k} \hat{u}$, $f^{(k)}:=\partial_{t}^{k} f$, see, for example, [19]. We shall make use of these estimates in the sequel. The error $\eta$ of the interpolant $L^{n} \circ \pi_{h}^{n} \varepsilon$ of $\varepsilon=\hat{u}-u_{h}$ will result in high order space-time derivatives of the error $\varepsilon$. The restriction of $\partial_{t}^{q} \partial_{x}^{\mu} \varepsilon$ to an element of the partition may be written as $\partial_{t}^{q} \partial_{x}^{\mu} \hat{u}-\partial_{t}^{q} \partial_{x}^{\mu} u_{h}$. We shall estimate the continuous solution derivatives $\partial_{t}^{q} \partial_{x}^{\mu} \hat{u}$ by $(3.12)$ while $\partial_{t}^{q} \partial_{x}^{\mu} u_{h}$ will be used in the estimator.

In view of (3.7), we shall need to control the terms $\left|\eta^{i+0}\right|_{\hat{\Omega}},\|\eta\|_{L^{2}(K)}$, and $\|\eta\|_{L^{2}(\ell)}$, so as to establish an $a$ posteriori estimate. This is accomplished in the next two lemmata assuming a regular partition in $t$, i.e., that $\frac{k_{i}}{k_{j}} \geq c$ for any $i, j$, where $c$ is some positive constant.

Lemma 3.4. For any $1 \leq n \leq N$, it holds that

$$
\begin{aligned}
& \sum_{i=0}^{n-1}\left|\eta^{i+0}\right|_{\hat{\Omega}}^{2} \leq c \boldsymbol{h}_{n}^{2} \boldsymbol{k}_{n}^{-1} \sum_{i=0}^{n-1} \sum_{K \in T_{h}^{i}} \int_{I_{i}}\|\varepsilon\|_{H^{1}(K)}^{2} d t+c \boldsymbol{h}_{n}^{2} \boldsymbol{k}_{n} \sum_{i=0}^{n-1} \sum_{K \in T_{h}^{i}} \int_{I_{i}}\left\|u_{h t}\right\|_{H^{1}(K)}^{2} d t \\
& +c \boldsymbol{k}_{n}^{2 q-1} \boldsymbol{h}_{n}^{2}\left[\left\|\hat{u}^{0}\right\|_{H^{2 q+1}(\hat{\Omega})}^{2}+\sum_{k=0}^{q}\left\|\hat{f}^{(k)}\right\|_{L^{2}\left(0, t^{n} ; H^{2 q-2 k}(\hat{\Omega})\right)}^{2}+\sum_{i=0}^{n-1} \sum_{K \in T_{h}^{i}} \int_{I_{i}}\left\|u_{h}^{(q)}\right\|_{H^{1}(K)}^{2} d t\right] \\
& +c \boldsymbol{k}_{n}^{2 q+1} \boldsymbol{h}_{n}^{2}\left[\left\|\hat{u}^{0}\right\|_{H^{2 q+3}(\hat{\Omega})}^{2}+\sum_{k=0}^{q+1}\left\|\hat{f}^{(k)}\right\|_{L^{2}\left(0, t^{n} ; H^{2 q+2-2 k}(\hat{\Omega})\right)}^{2}+\sum_{i=0}^{n-1} \sum_{K \in T_{h}^{i}} \int_{I_{i}}\left\|u_{h}^{(q+1)}\right\|_{H^{1}(K)}^{2} d t\right] \\
& +c \boldsymbol{k}_{n}^{2 q-1}\left[\left\|\hat{u}^{0}\right\|_{H^{2 q-1}(\hat{\Omega})}^{2}+\sum_{k=0}^{q-1}\left\|\hat{f}^{(k)}\right\|_{L^{2}\left(0, t^{n} ; H^{2 q-2-2 k}(\hat{\Omega})\right)}+\sum_{i=0}^{n-1} \sum_{K \in T_{h}^{i}} \int_{I_{i}}\left\|u_{h}^{(q)}\right\|_{L^{2}(K)}^{2} d t\right] \\
& +c \boldsymbol{k}_{n}^{2 q+1}\left[\left\|\hat{u}^{0}\right\|_{H^{2 q+1}(\hat{\Omega})}^{2}+\sum_{k=0}^{q}\left\|\hat{f}^{(k)}\right\|_{L^{2}\left(0, t^{n} ; H^{2 q-2 k}(\hat{\Omega})\right)}+\sum_{i=0}^{n-1} \sum_{K \in T_{h}^{i}} \int_{I_{i}}\left\|u_{h}^{(q+1)}\right\|_{L^{2}(K)}^{2} d t\right] \\
& +c \boldsymbol{h}_{n}^{2} \boldsymbol{k}_{n}\left[\left\|\hat{u}^{0}\right\|_{H^{3}(\hat{\Omega})}^{2}+\sum_{k=0}^{1}\left\|\hat{f}^{(k)}\right\|_{L^{2}\left(0, t^{n} ; H^{2-2 k}(\hat{\Omega})\right)}^{2}\right],
\end{aligned}
$$

Proof. Observe first that for $g$ smooth in $t$ it follows that [4]

$$
\max _{t \in \overline{I_{i}}}\left|L^{i}(g)-g\right| \leq c k_{i}^{q} \max _{t \in \bar{I}_{i}}\left|g^{(q)}(\cdot, t)\right| .
$$


Furthermore, if $v$ is smooth then $\left(\pi_{h}^{i} v\right)^{(q)}=\pi_{h}^{i}\left(v^{(q)}\right)$. Indeed, $\operatorname{dim} \hat{\Omega}=2$, and the domain is rectangular, so $\pi_{h}^{i}$ is the standard Clemént interpolant defined via the local $L^{2}$ projections in space. Thus, the operator $\pi_{h}^{i}$ is independent of $t$ for any $i$, since $\hat{\Omega}$ is time independent. We write

$$
\eta^{i+0}=\varepsilon\left(\cdot, t^{i+0}\right)-L^{i} \circ \pi_{h}^{i} \varepsilon\left(\cdot, t^{i+0}\right)=\varepsilon\left(\cdot, t^{i+0}\right)-\pi_{h}^{i} \varepsilon\left(\cdot, t^{i+0}\right)-\left(L^{i}-I\right) \pi_{h}^{i} \varepsilon\left(\cdot, t^{i+0}\right),
$$

and use the previous estimates together with (3.11) and the local Sobolev inequality 5.3 for $t \in I_{i}$, to obtain

$$
\left|\eta^{i+0}\right|_{\hat{\Omega}}^{2} \leq c \boldsymbol{h}_{n}^{2} \sum_{K \in T_{h}^{i}} \int_{I_{i}}\left[k_{i}^{-1}\|\varepsilon\|_{H^{1}(\Delta K)}^{2}+k_{i}\left\|\varepsilon_{t}\right\|_{H^{1}(\Delta K)}^{2}\right] d t+c k_{i}^{2 q} \int_{0}^{1} \int_{\theta_{1}}^{\theta_{2}}\left(\max _{t \in \bar{I}_{i}}\left|\pi_{h}^{i}\left(\varepsilon^{(q)}\right)(\cdot, t)\right|\right)^{2} d \theta d y .
$$

Using again (5.3), we get

$$
\begin{aligned}
\left|\pi_{h}^{i}\left(\varepsilon^{(q)}\right)(t)\right| \leq\left|\pi_{h}^{i}\left(\varepsilon^{(q)}\right)(t)-\varepsilon^{(q)}(t)\right|+\left|\varepsilon^{(q)}(t)\right| \leq & c\left(k_{i}^{-1} \int_{I_{i}}\left[\left|\pi_{h}^{i}\left(\varepsilon^{(q)}\right)-\varepsilon^{(q)}\right|^{2}+\left|\varepsilon^{(q)}\right|^{2}\right] d t\right)^{1 / 2} \\
& +c\left(k_{i} \int_{I_{i}}\left[\left|\pi_{h}^{i}\left(\varepsilon^{(q+1)}\right)-\varepsilon^{(q+1)}\right|^{2}+\left|\varepsilon^{(q+1)}\right|^{2}\right] d t\right)^{1 / 2} .
\end{aligned}
$$

Splitting the time derivatives of $\varepsilon=\hat{u}-u_{h}$ and using the above inequalities, we obtain

$$
\begin{aligned}
\left|\eta^{i+0}\right|_{\hat{\Omega}}^{2} \leq & c \boldsymbol{h}_{n}^{2} k_{i}^{-1} \sum_{K \in T_{h}^{i}} \int_{I_{i}}\|\varepsilon\|_{H^{1}(K)}^{2} d t+c \boldsymbol{h}_{n}^{2} k_{i} \sum_{K \in T_{h}^{i}} \int_{I_{i}}\left\|u_{h t}\right\|_{H^{1}(K)}^{2} d t \\
& +c k_{i}^{2 q-1} \boldsymbol{h}_{n}^{2} \sum_{K \in T_{h}^{i}} \int_{I_{i}}\left\|u_{h}^{(q)}\right\|_{H^{1}(K)}^{2} d t+c k_{i}^{2 q+1} \boldsymbol{h}_{n}^{2} \sum_{K \in T_{h}^{i}} \int_{I_{i}}\left\|u_{h}^{(q+1)}\right\|_{H^{1}(K)}^{2} d t \\
& +c k_{i}^{2 q-1} \sum_{K \in T_{h}^{i}} \int_{I_{i}}\left\|u_{h}^{(q)}\right\|_{L^{2}(K)}^{2} d t+c k_{i}^{2 q+1} \sum_{K \in T_{h}^{i}} \int_{I_{i}}\left\|u_{h}^{(q+1)}\right\|_{L^{2}(K)}^{2} d t+\mathcal{A}
\end{aligned}
$$

where

$$
\begin{aligned}
\mathcal{A} \leq & c \boldsymbol{h}_{n}^{2} k_{i} \sum_{K \in T_{h}^{i}} \int_{I_{i}}\left\|\hat{u}_{t}\right\|_{H^{1}(K)}^{2} d t+c k_{i}^{2 q-1} \boldsymbol{h}_{n}^{2} \sum_{K \in T_{h}^{i}} \int_{I_{i}}\left\|\hat{u}^{(q)}\right\|_{H^{1}(K)}^{2} d t+c k_{i}^{2 q+1} \boldsymbol{h}_{n}^{2} \sum_{K \in T_{h}^{i}} \int_{I_{i}}\left\|\hat{u}^{(q+1)}\right\|_{H^{1}(K)}^{2} d t \\
& +c k_{i}^{2 q-1} \sum_{K \in T_{h}^{i}} \int_{I_{i}}\left\|\hat{u}^{(q)}\right\|_{L^{2}(K)}^{2} d t+c k_{i}^{2 q+1} \sum_{K \in T_{h}^{i}} \int_{I_{i}}\left\|\hat{u}^{(q+1)}\right\|_{L^{2}(K)}^{2} d t .
\end{aligned}
$$

Summing over $i$ and applying the estimates 3.12 for the time derivatives of $\hat{u}$ we obtain 3.13 . 
Lemma 3.5. For any $1 \leq n \leq N$, it holds that

$$
\begin{aligned}
\sum_{i=0}^{n-1} \sum_{K \in T_{h}^{i}} \int_{I_{i}}\|\eta\|_{L^{2}(K)}^{2} d t \leq & c \boldsymbol{h}_{n}^{2}\left(\sum_{i=0}^{n-1} \sum_{K \in T_{h}^{i}} \int_{I_{i}}\|\varepsilon\|_{L^{2}(K)}^{2} d t+\sum_{i=0}^{n-1} \sum_{K \in T_{h}^{i}} \int_{I_{i}}\|\nabla \varepsilon\|_{L^{2}(K)}^{2} d t\right) \\
& +c \boldsymbol{k}_{n}^{2 q}\left[\left\|\hat{u}^{0}\right\|_{H^{2 q-1}(\hat{\Omega})}^{2} \sum_{k=0}^{q-1}\left\|\hat{f}^{(k)}\right\|_{\left.L^{2}\left(0, t^{n} ; H^{2 q-2-2 k}(\hat{\Omega})\right)\right]}^{2}\right. \\
& +c \boldsymbol{k}_{n}^{2 q+2}\left[\left\|\hat{u}^{0}\right\|_{H^{2 q+1}(\hat{\Omega})}^{2}+\sum_{k=0}^{q}\left\|\hat{f}^{(k)}\right\|_{L^{2}\left(0, t^{n} ; H^{2 q-2 k}(\hat{\Omega})\right)}^{2}\right] \\
& +c \boldsymbol{k}_{n}^{2 q} \sum_{i=0}^{n-1} \sum_{K \in T_{h}^{i}} \int_{I_{i}}\left\|u_{h}^{(q)}\right\|_{L^{2}(K)}^{2} d t \\
& +c \boldsymbol{k}_{n}^{2 q+2} \sum_{i=0}^{n-1} \sum_{K \in T_{h}^{i}} \int_{I_{i}}\left\|u_{h}^{(q+1)}\right\|_{L^{2}(K)}^{2} d t,
\end{aligned}
$$

and

$$
\begin{aligned}
\sum_{i=0}^{n-1} \sum_{K \in T_{h}^{i}} \sum_{\ell \in E_{K}^{i}} \int_{I_{i}}\|\eta\|_{L^{2}(\ell)}^{2} d t \leq & c \boldsymbol{h}_{n}\left(\sum_{i=0}^{n-1} \sum_{K \in T_{h}^{i}} \int_{I_{i}}\|\varepsilon\|_{L^{2}(K)}^{2} d t+\sum_{i=0}^{n-1} \sum_{K \in T_{h}^{i}} \int_{I_{i}}\|\nabla \varepsilon\|_{L^{2}(K)}^{2} d t\right) \\
& +c \boldsymbol{k}_{n}^{2 q} \boldsymbol{h}_{n}^{-1}\left[\left\|\hat{u}^{0}\right\|_{H^{2 q+1}(\hat{\Omega})}^{2}+\sum_{k=0}^{q}\left\|\hat{f}^{(k)}\right\|_{\left.L^{2}\left(0, t^{n} ; H^{2 q-2 k}(\hat{\Omega})\right)\right]}^{2}\right] \\
& +c \boldsymbol{k}_{n}^{2 q+2} \boldsymbol{h}_{n}^{-1}\left[c\left\|\hat{u}^{0}\right\|_{H^{2 q+3}(\hat{\Omega})}^{2}+c \sum_{k=0}^{q+1}\left\|\hat{f}^{(k)}\right\|_{\left.L^{2}\left(0, t^{n} ; H^{2 q+2-2 k}(\hat{\Omega})\right)\right]}^{2}\right] \\
& +c \boldsymbol{k}_{n}^{2 q} \boldsymbol{h}_{n}^{-1} \sum_{i=0}^{n-1} \sum_{K \in T_{h}^{i}} \sum_{\ell \in E_{K}^{i}} \int_{I_{i}}\left\|u_{h}^{(q)}\right\|_{L^{2}(\ell)}^{2} d t \\
& +c \boldsymbol{k}_{n}^{2 q+2} \boldsymbol{h}_{n}^{-1} \sum_{i=0}^{n-1} \sum_{K \in T_{h}^{i}} \sum_{\ell \in E_{K}^{i}} \int_{I_{i}}\left\|u_{h}^{(q+1)}\right\|_{L^{2}(\ell)}^{2} d t
\end{aligned}
$$

Proof. By 3.9 and using the fact that $L^{i}$ is the $L^{2}$ projection, we have for $\mathcal{A}=K, \ell$,

$$
\begin{aligned}
\int_{I_{i}}\|\eta\|_{L^{2}(\mathcal{A})}^{2} d t & \leq 2 \int_{I_{i}}\left\|\varepsilon-L^{i} \varepsilon\right\|_{L^{2}(\mathcal{A})}^{2} d t+2 \int_{I_{i}}\left\|L^{i} \varepsilon-L^{i} \circ \pi_{h}^{i} \varepsilon\right\|_{L^{2}(\mathcal{A})}^{2} d t \\
& \leq 2 k_{i}^{2 q+1}\left\|\max _{t \in \bar{I}_{i}}\left|\varepsilon^{(q)}(\cdot, t)\right|\right\|_{L^{2}(\mathcal{A})}^{2}+2 \int_{I_{i}}\left\|\varepsilon-\pi_{h}^{i} \varepsilon\right\|_{L^{2}(\mathcal{A})}^{2} d t \\
& \leq 2 k_{i}^{2 q+1}\left\|\max _{t \in \bar{I}_{i}}\left|\varepsilon^{(q)}(\cdot, t)\right|\right\|_{L^{2}(\mathcal{A})}^{2}+c h_{\mathcal{A}}^{2}\left(2 \int_{I_{i}}\|\varepsilon\|_{L^{2}(\Delta K)}^{2} d t+2 \int_{I_{i}}\|\nabla \varepsilon\|_{L^{2}(\Delta K)}^{2} d t\right),
\end{aligned}
$$

where $h_{\mathcal{A}}=\boldsymbol{h}_{n}$ if $\mathcal{A}=K$, or $h_{\mathcal{A}}=\boldsymbol{h}_{n}^{1 / 2}$ if $\mathcal{A}=\ell$. Note that by a local Sobolev inequality in $\mathbb{R}(\mathrm{cf}$. $5.3 \mathrm{p}$ ) it holds that

$$
\left.\max _{t \in \bar{I}_{i}}\left|\varepsilon^{(q)}(\cdot, t)\right|^{2} \leq c k_{i}^{-1} \int_{I_{i}}\left|\varepsilon^{(q)}(\cdot, t)\right|^{2} d t+c k_{i} \int_{I_{i}}\left|\varepsilon^{(q+1)}(\cdot, t)\right|^{2}\right) d t
$$


and thus

$$
\begin{aligned}
\left\|\max _{t \in \bar{I}_{i}}\left|\varepsilon^{(q)}(\cdot, t)\right|\right\|_{L^{2}(\mathcal{A})}^{2} \leq & c k_{i}^{-1} \int_{I_{i}}\left\|\hat{u}^{(q)}\right\|_{L^{2}(\mathcal{A})}^{2} d t+c k_{i}^{-1} \int_{I_{i}}\left\|u_{h}^{(q)}\right\|_{L^{2}(\mathcal{A})}^{2} d t \\
& +c k_{i} \int_{I_{i}}\left\|\hat{u}^{(q+1)}\right\|_{L^{2}(\mathcal{A})}^{2} d t+c k_{i} \int_{I_{i}}\left\|u_{h}^{(q+1)}\right\|_{L^{2}(\mathcal{A})}^{2} d t .
\end{aligned}
$$

Substituting in 3.16$)$ we obtain

$$
\begin{aligned}
\int_{I_{i}}\|\eta\|_{L^{2}(K)}^{2} d t \leq & 2 c k_{i}^{2 q} \int_{I_{i}}\left\|\hat{u}^{(q)}\right\|_{L^{2}(K)}^{2} d t+2 c k_{i}^{2 q} \int_{I_{i}}\left\|u_{h}^{(q)}\right\|_{L^{2}(K)}^{2} d t \\
& +2 c k_{i}^{2 q+2} \int_{I_{i}}\left\|\hat{u}^{(q+1)}\right\|_{L^{2}(K)}^{2} d t+2 c k_{i}^{2 q+2} \int_{I_{i}}\left\|u_{h}^{(q+1)}\right\|_{L^{2}(K)}^{2} d t \\
& +c \boldsymbol{h}_{n}^{2}\left(2 \int_{I_{i}}\|\varepsilon\|_{L^{2}(\Delta K)}^{2} d t+2 \int_{I_{i}}\|\nabla \varepsilon\|_{L^{2}(\Delta K)}^{2} d t\right) .
\end{aligned}
$$

Using the local trace inequality (5.1)

$$
\left\|\hat{u}^{(q)}\right\|_{L^{2}(\ell)}^{2} \leq \int_{\partial K}\left|\hat{u}^{(q)}\right|^{2} d s \leq c \boldsymbol{h}_{n}^{-1}\left\|\hat{u}^{(q)}\right\|_{H^{1}(K)}^{2},
$$

we obtain

$$
\begin{aligned}
\int_{I_{i}}\|\eta\|_{L^{2}(\ell)}^{2} d t \leq & 2 c k_{i}^{2 q} \boldsymbol{h}_{n}^{-1} \int_{I_{i}}\left\|\hat{u}^{(q)}\right\|_{H^{1}(K)}^{2} d t+2 c k_{i}^{2 q} \boldsymbol{h}_{n}^{-1} \int_{I_{i}}\left\|u_{h}^{(q)}\right\|_{L^{2}(\ell)}^{2} d t \\
& +2 c k_{i}^{2 q+2} \boldsymbol{h}_{n}^{-1} \int_{I_{i}}\left\|\hat{u}^{(q+1)}\right\|_{H^{1}(K)}^{2} d t+2 c k_{i}^{2 q+2} \boldsymbol{h}_{n}^{-1} \int_{I_{i}}\left\|u_{h}^{(q+1)}\right\|_{L^{2}(\ell)}^{2} d t \\
& +c \boldsymbol{h}_{n}\left(2 \int_{I_{i}}\|\varepsilon\|_{L^{2}(\Delta K)}^{2} d t+2 \int_{I_{i}}\|\nabla \varepsilon\|_{L^{2}(\Delta K)}^{2} d t\right) .
\end{aligned}
$$

Using (3.12) we arrive at

$$
\begin{aligned}
& \sum_{i=0}^{n-1} \sum_{K \in T_{h}^{i}} \int_{I_{i}}\left\|\hat{u}^{(q)}\right\|_{L^{2}(K)}^{2} \leq\left\|\hat{u}^{(q)}\right\|_{L^{2}\left(0, t^{n} ; L^{2}(\hat{\Omega})\right)}^{2} \leq c\left\|\hat{u}^{0}\right\|_{H^{2 q-1}(\hat{\Omega})}^{2}+c \sum_{k=0}^{q-1}\left\|\hat{f}^{(k)}\right\|_{L^{2}\left(0, t^{n} ; H^{2 q-2-2 k}(\hat{\Omega})\right)}^{2}, \\
& \sum_{i=0}^{n-1} \sum_{K \in T_{h}^{i}} \int_{I_{i}}\left\|\hat{u}^{(q+1)}\right\|_{L^{2}(K)}^{2} \leq\left\|\hat{u}^{(q+1)}\right\|_{L^{2}\left(0, t^{n} ; L^{2}(\hat{\Omega})\right)}^{2} \leq c\left\|\hat{u}^{0}\right\|_{H^{2 q+1}(\hat{\Omega})}^{2}+c \sum_{k=0}^{q}\left\|\hat{f}^{(k)}\right\|_{L^{2}\left(0, t^{n} ; H^{2 q-2 k}(\hat{\Omega})\right)}^{2},
\end{aligned}
$$

while

$$
\sum_{i=0}^{n-1} \sum_{K \in T_{h}^{i}} \sum_{\ell \in E_{K}^{i}} \int_{I_{i}}\left\|\hat{u}^{(q)}\right\|_{H^{1}(K)}^{2} \leq\left\|\hat{u}^{(q)}\right\|_{L^{2}\left(0, t^{n} ; H^{1}(\hat{\Omega})\right)}^{2} \leq c\left\|\hat{u}^{0}\right\|_{H^{2 q+1}(\hat{\Omega})}^{2}+c \sum_{k=0}^{q}\left\|\hat{f}^{(k)}\right\|_{L^{2}\left(0, t^{n} ; H^{2 q-2 k}(\hat{\Omega})\right)}^{2},
$$

and

$$
\begin{aligned}
& \sum_{i=0}^{n-1} \sum_{K \in T_{h}^{i}} \sum_{\ell \in E_{K}^{i}} \int_{I_{i}}\left\|\hat{u}^{(q+1)}\right\|_{H^{1}(K)}^{2} d t \leq\left\|\hat{u}^{(q+1)}\right\|_{L^{2}\left(0, t^{n} ; H^{1}(\hat{\Omega})\right)}^{2} \\
& \quad \leq c\left\|\hat{u}^{0}\right\|_{H^{2 q+3}(\hat{\Omega})}^{2}+c \sum_{k=0}^{q+1}\left\|\hat{f}^{(k)}\right\|_{L^{2}\left(0, t^{n} ; H^{2 q+2-2 k}(\hat{\Omega})\right)}^{2} .
\end{aligned}
$$

Summing over $i$ and $K$ in (3.18) and summing over $i, K, \ell$ in (3.19), and by substituting in (3.20) and (3.21), respectively, we obtain the estimates (3.14) and (3.15). 
The following theorem presents an upper bound for the a posteriori error. Its proof follows by using in (3.7) the fact that $\beta_{1}$ may be suitably chosen and the estimates (3.13), (3.14) and (3.15).

Theorem 3.6. If the solution $\hat{u}$ of problem (3.1), satisfies (3.2) for $k, \mu=0, \ldots, m+1$, for $m=q$, and if $\boldsymbol{h}_{n}^{2} \boldsymbol{k}_{n}^{-1}$ is sufficiently small, then the error $\varepsilon=\hat{u}-u_{h}$ satisfies

$$
\begin{aligned}
& \sum_{i=0}^{n-1} \sum_{K \in T_{h}^{i}}\left\{\|\varepsilon\|_{K}^{2}+\|\nabla \varepsilon\|_{K}^{2}\right\}+\left|\varepsilon^{n}\right|_{\hat{\Omega}}^{2}+\sum_{i=0}^{n-1}\left|u_{h}^{i+0}-u_{h}^{i}\right|_{\hat{\Omega}}^{2} \leq \\
& c\left|\varepsilon^{0}\right|_{\hat{\Omega}}^{2}+\left[c \boldsymbol{h}_{n}^{2}\right] \sum_{i=0}^{n-1} \sum_{K \in T_{h}^{i}}\left\|\hat{f}-\partial_{t} u_{h}+A u_{h y y}+B u_{h y \theta}+C u_{h \theta \theta}+D u_{h y}+E u_{h \theta}+\beta_{1} u_{h}\right\|_{L^{2}(K)}^{2} \\
& +\left[c \boldsymbol{h}_{n}\right] \sum_{i=0}^{n-1} \sum_{K \in T_{h}^{i}} \sum_{\ell \in E_{K}^{i}} \int_{\ell}\left|\left[\mathcal{M} \nabla u_{h} \cdot \boldsymbol{n}\right]_{\ell}\right|^{2} d s \\
& +\left[c \boldsymbol{k}_{n}^{2 q} \boldsymbol{h}_{n}^{-2}\right] \sum_{i=0}^{n-1} \sum_{K \in T_{h}^{i}} \sum_{\ell \in E_{K}^{i}} \int_{I_{i}}\left\|u_{h}^{(q)}\right\|_{L^{2}(\ell)}^{2}+\left[c \boldsymbol{k}_{n}^{2 q+2} \boldsymbol{h}_{n}^{-2}\right] \sum_{i=0}^{n-1} \sum_{K \in T_{h}^{i}} \sum_{\ell \in E_{K}^{i}} \int_{I_{i}}\left\|u_{h}^{(q+1)}\right\|_{L^{2}(\ell)}^{2} d t \\
& +\left[c \boldsymbol{h}_{n}^{2} \boldsymbol{k}_{n}\right] \mathcal{E}_{1}+\left[c \boldsymbol{k}_{n}^{2 q-1} \boldsymbol{h}_{n}^{2}\right] \mathcal{E}_{2}+\left[c \boldsymbol{k}_{n}^{2 q+1} \boldsymbol{h}_{n}^{2}\right] \mathcal{E}_{3}+\left[c \boldsymbol{k}_{n}^{2 q-1}\right] \mathcal{E}_{4} \\
& +\left[c \boldsymbol{k}_{n}^{2 q+1}\right] \mathcal{E}_{5}+\left[c \boldsymbol{k}_{n}^{2 q} \boldsymbol{h}_{n}^{-2}\right] \mathcal{E}_{6}+\left[c \boldsymbol{k}_{n}^{2 q+2} \boldsymbol{h}_{n}^{-2}\right] \mathcal{E}_{7},
\end{aligned}
$$

where $\mathcal{E}_{i}=\mathcal{E}_{i}\left(\hat{u}^{0}, \hat{f}, u_{h}\right), i=1, \cdots, 7$, are given by

$$
\begin{gathered}
\mathcal{E}_{1}:=\left[\sum_{i=0}^{n-1} \sum_{K \in T_{h}^{i}} \int_{I_{i}}\left\|u_{h t}\right\|_{H^{1}(K)}^{2} d t+\left\|\hat{u}^{0}\right\|_{H^{3}(\hat{\Omega})}^{2}+\sum_{k=0}^{1}\left\|\hat{f}^{(k)}\right\|_{L^{2}\left(0, t^{n} ; H^{2-2 k}(\hat{\Omega})\right)}^{2}\right], \\
\mathcal{E}_{2}:=\left[\left\|\hat{u}^{0}\right\|_{H^{2 q+1}(\hat{\Omega})}^{2}+\sum_{k=0}^{q}\left\|\hat{f}^{(k)}\right\|_{L^{2}\left(0, t^{n} ; H^{2 q-2 k}(\hat{\Omega})\right)}^{2}+\sum_{i=0}^{n-1} \sum_{K \in T_{h}^{i}} \int_{I_{i}}\left\|u_{h}^{(q)}\right\|_{H^{1}(K)}^{2} d t\right], \\
\mathcal{E}_{3}:=\left[\left\|\hat{u}^{0}\right\|_{H^{2 q+3}(\hat{\Omega})}^{2}+\sum_{k=0}^{q+1}\left\|\hat{f}^{(k)}\right\|_{L^{2}\left(0, t^{n} ; H^{2 q+2-2 k}(\hat{\Omega})\right)}^{2}+\sum_{i=0}^{n-1} \sum_{K \in T_{h}^{i}} \int_{I_{i}}\left\|u_{h}^{(q+1)}\right\|_{H^{1}(K)}^{2} d t\right], \\
\mathcal{E}_{4}:=\left[\left\|\hat{u}^{0}\right\|_{H^{2 q-1}(\hat{\Omega})}^{2}+\sum_{k=0}^{q-1}\left\|\hat{f}^{(k)}\right\|_{L^{2}\left(0, t^{n} ; H^{2 q-2-2 k}(\hat{\Omega})\right)}+\sum_{i=0}^{n-1} \sum_{K \in T_{h}^{i}} \int_{I_{i}}\left\|u_{h}^{(q)}\right\|_{L^{2}(K)}^{2} d t\right], \\
\mathcal{E}_{5}:=\left[\left\|\hat{u}^{0}\right\|_{H^{2 q+1}(\hat{\Omega})}^{2}+\sum_{k=0}^{q-1}\left\|\hat{f}^{(k)}\right\|_{L^{2}\left(0, t^{n} ; H^{2 q-2 k}(\hat{\Omega})\right)}+\sum_{i=0} \sum_{K \in T_{h}^{i}} \int_{I_{i}}\left\|u_{h}^{(q+1)}\right\|_{L^{2}(K)}^{2} d t\right], \\
\left.\quad+\left\|\hat{u}^{0}\right\|_{H^{2 q+1}(\hat{\Omega})}^{2}+\sum_{k=0}^{q}\left\|\hat{f}^{(k)}\right\|_{L^{2}\left(0, t^{n} ; H^{2 q-2 k}(\hat{\Omega})\right)}^{2}\right] \\
\mathcal{E}_{6}:=\left[\left\|\hat{u}^{0}\right\|_{H^{2 q-1}(\hat{\Omega})}^{q-1}\left\|\hat{f}_{k=0}^{(k)}\right\|_{L^{2}\left(0, t^{n} ; H^{2 q-2-2 k}(\hat{\Omega})\right)}^{n-1}+\sum_{i=0} \sum_{K \in T_{h}^{i}} \int_{I_{i}}\left\|u_{h}^{(q)}\right\|_{L^{2}(K)}^{2}\right.
\end{gathered}
$$




$$
\begin{aligned}
\mathcal{E}_{7}:=\quad\left[\left\|\hat{u}^{0}\right\|_{H^{2 q+1}(\hat{\Omega})}^{2}+\sum_{k=0}^{q}\left\|\hat{f}^{(k)}\right\|_{L^{2}\left(0, t^{n} ; H^{2 q-2 k}(\hat{\Omega})\right)}^{2}+\sum_{i=0}^{n-1} \sum_{K \in T_{h}^{i}} \int_{I_{i}}\left\|u_{h}^{(q+1)}\right\|_{L^{2}(K)}^{2} d t\right. \\
\left.+\left\|\hat{u}^{0}\right\|_{H^{2 q+3}(\hat{\Omega})}^{2}+\sum_{k=0}^{q+1}\left\|\hat{f}^{(k)}\right\|_{L^{2}\left(0, t^{n} ; H^{2 q+2-2 k}(\hat{\Omega})\right)}^{2}\right],
\end{aligned}
$$

for any $1 \leq n \leq N$. It is assumed that $\hat{f}^{(k)} \in L^{2}\left(0, s ; H^{2 m-2 k}(\hat{\Omega})\right)$ for any $k=0, \ldots, m+1, m=q-1, q, q+1$, and that $\hat{u}^{0} \in H^{2 q+3}(\hat{\Omega})$.

Several remarks concerning the nature of this estimate are in order:

1. The assumption $\boldsymbol{h}_{n}^{2} \boldsymbol{k}_{n}^{-1} \leq c$, when $\mathcal{O}\left(\boldsymbol{h}_{n}\right)=\mathcal{O}\left(\boldsymbol{k}_{n}\right)$, is transformed into a smallness assumption for the space discretization parameter $\boldsymbol{h}_{n}$.

2. The estimate of Theorem 3.6 involves the $L^{2}$ and $H^{1}$ norms of the error $\varepsilon$. Note that since $m=2$, the lengths of the interior edges $|\ell|$ are of order $\mathcal{O}\left(\boldsymbol{h}_{n}\right)$ while the areas of the elements $|K|$ of the partition are of order $\mathcal{O}\left(\boldsymbol{h}_{n}^{2}\right)$. Thus, the a posteriori estimate of Theorem 3.6 is optimal in space, as an $H^{1}$ estimate, if the $\|\cdot\|_{L^{2}(K)}^{2},\|\cdot\|_{H^{1}(K)}^{2}$ coefficients are of order at least $\mathcal{O}\left(\boldsymbol{h}_{n}^{2}\right)$, and if the $\|\cdot\|_{L^{2}(\ell)}^{2}$ and trace term coefficients involving $\mathcal{M}$ are of order at least $\mathcal{O}\left(\boldsymbol{h}_{n}\right)$. So, if $\mathcal{O}\left(\boldsymbol{k}_{n}\right)=\mathcal{O}\left(\boldsymbol{h}_{n}\right)$, then $q \geq 2$ provides an optimal result in $H^{1}$. However, if $\mathcal{O}\left(\boldsymbol{k}_{n}\right)=\mathcal{O}\left(\boldsymbol{h}_{n}\right)$, and $q \geq 2$, the estimate of Theorem 3.6 is sub-optimal in space as an $L^{2}$ estimate, and of total order $\boldsymbol{h}_{n}$, due to the trace terms. We have not estimated the constants appearing in the upper bound. However, attention must be given to the Gronwall constants that multiply the error terms $\mathcal{E}_{i}$ that iinvolve $\hat{f}$ and $\hat{u}^{0}$, for they may be large.

3. Assume that $\mathcal{O}\left(\boldsymbol{k}_{n}\right)=\mathcal{O}\left(\boldsymbol{h}_{n}\right)$. The aforementioned constants appear as coefficients of the terms involving $c \boldsymbol{h}_{n}^{3}, c \boldsymbol{h}_{n}^{2 q+1}, c \boldsymbol{h}_{n}^{2 q+3}, c \boldsymbol{h}_{n}^{2 q-1}, c \boldsymbol{h}_{n}^{2 q+1}, c \boldsymbol{h}_{n}^{2 q-2}, c \boldsymbol{h}_{n}^{2 q}$. If we select $q \geq 3$ then, he lowest order term is of order at least $\mathcal{O}\left(\boldsymbol{h}_{n}^{3}\right)$. Hence, for $\boldsymbol{h}_{n}$ small, all these coefficients are less than $c \boldsymbol{h}_{n}^{3}<1 \cdot \boldsymbol{h}_{n}^{2}$. Thus, the error estimate remains optimal in space in $H^{1}$ and the resulting a posteriori constants are controlled by the constant one.

4. When $m \geq 3$, our analysis may be easily extended to space-time, discontinuous in time Galerkin methods applied to parabolic initial and boundary value problems of general type, with Dirichlet boundary conditions, such as

$$
\begin{aligned}
& \hat{u}_{t}=\mathcal{L}(\hat{u})+F \text { in }(0, T) \times \tilde{\Omega}, \\
& \hat{u}(\cdot, 0)=\hat{u}^{0}, \\
& \hat{u}=0 \text { on } \partial \tilde{\Omega},
\end{aligned}
$$

for $\tilde{\Omega}$ a bounded domain in $\mathbb{R}^{m}$ with $m \geq 3$, and $\mathcal{L}(\hat{u})$ a second order elliptic operator with sufficiently smooth coefficients, provided that the space-time function $F$ and $\hat{u}^{0}$ are sufficiently smooth also.

3.3. Lower bound. We multiply the equation of the transformed problem (3.1) with some function $w$ in $H_{0}^{1}((0, T) \times \hat{\Omega})$ to be specified in the sequel and integrate in space-time so that

$$
\left(\left(\partial_{t} u, w\right)\right)_{\hat{G}^{n}}=\int_{t^{n}}^{t^{n+1}} \mathcal{B}(\hat{u}, w) d t+\left(\left(\widetilde{D} \hat{u}_{y}+\widetilde{E} \hat{u}_{\theta}+\beta_{1} \hat{u}, w\right)\right)_{\hat{G}^{n}}+((\hat{f}, w))_{\hat{G}^{n}}
$$

Writing $\hat{u}=\hat{u}-u_{h}+u_{h}=\varepsilon+u_{h}$ we get

$$
\begin{aligned}
& \left(\left(\partial_{t} \varepsilon, w\right)\right)_{\hat{G}^{n}}-\int_{t^{n}}^{t^{n+1}} \mathcal{B}(\varepsilon, w) d t-\left(\left(\widetilde{D} \varepsilon_{y}+\widetilde{E} \varepsilon_{\theta}+\beta_{1} \varepsilon, w\right)\right)_{\hat{G}^{n}}= \\
& \sum_{K \in T_{h}^{n}}\left(\left(-\partial_{t} u_{h}+\hat{f}+A u_{h y y}+B u_{h y \theta}+C u_{h \theta \theta}+D u_{h y}+E u_{h \theta}+\beta_{1} u_{h}, w\right)\right)_{I_{n} \times K} \\
& -\int_{I_{n}} \sum_{K \in T_{h}^{n}} \sum_{\ell \in E_{K}^{n}} \int_{\ell} w\left[\mathcal{M} \nabla u_{h} \cdot \mathbf{n}\right]_{\ell} d s d t .
\end{aligned}
$$


Following the arguments of [8, 43, 36] and assume that the meshes space-time meshes are regular with a regularity constant $\beta_{\text {reg. }}$ Furthermore, we let

$$
A_{h}:=-\partial_{t} u_{h}+\hat{f}+A u_{h y y}+B u_{h y \theta}+C u_{h \theta \theta}+D u_{h y}+E u_{h \theta}+\beta_{1} u_{h}, \quad B_{h}:=-\left[\mathcal{M} \nabla u_{h} \cdot \mathbf{n}\right]_{\ell} .
$$

Then, there exists a constant $c$, depending on $\beta_{\text {reg, }}$ and a function $w \in H_{0}^{1}((0, T) \times \hat{\Omega})$ such that for all $n=0, \cdots, N-1$ and for all $K \in T_{h}^{n}$ it holds that (cf. 36] pp. 225, 229),

$$
\begin{gathered}
w\left(\cdot, t^{n}\right)=0, \\
|K| \int_{t^{n}}^{t^{n+1}}\left\|\Pi_{K}^{n}\left(A_{h}\right)\right\|_{L^{2}(K)}^{2} d t=\int_{t^{n}}^{t^{n+1}} \int_{K} \Pi_{K}^{n}\left(A_{h}\right) w d x d t, \\
|\ell| \int_{t^{n}}^{t^{n+1}}\left\|\Pi_{\ell}^{n}\left(B_{h}\right)\right\|_{L^{2}(\ell)}^{2} d t=\int_{t^{n}}^{t^{n+1}} \int_{\ell} \Pi_{\ell}^{n}\left(B_{h}\right) w d s d t \quad \forall \ell \in E_{K}^{n}, \\
|w|_{1,\left(I_{n} \times K\right)}^{2} \leq c \int_{t^{n}}^{t^{n+1}}\left\{|K|\left\|\Pi_{K}^{n}\left(A_{h}\right)\right\|_{L^{2}(K)}^{2}+\sum_{\ell \in E_{K}^{n}}|\ell|\left\|\Pi_{\ell}^{n}\left(B_{h}\right)\right\|_{L^{2}(\ell)}^{2}\right\} d t,
\end{gathered}
$$

where

$$
\begin{aligned}
\Pi_{k}^{n}(v) & :=\frac{1}{\left(t^{n+1}-t^{n}\right)|K|} \int_{t^{n}}^{t^{n+1}} \int_{K} v d x d t, \\
\Pi_{\ell}^{n}(v) & :=\frac{1}{\left(t^{n+1}-t^{n}\right)|\ell|} \int_{t^{n}}^{t^{n+1}} \int_{\ell} v d s d t .
\end{aligned}
$$

Here, $|w|_{1,\left(I_{n} \times K\right)}$ denotes the space-time seminorm. Let us now define

$$
\eta_{\mathrm{lb}}^{n}:=\left[\int_{t^{n}}^{t^{n+1}}\left\{|K|\left\|\Pi_{K}^{n}\left(A_{h}\right)\right\|_{L^{2}(K)}^{2}+\sum_{\ell \in E_{K}^{n}}|\ell|\left\|\Pi_{\ell}^{n}\left(B_{h}\right)\right\|_{L^{2}(\ell)}^{2}\right\} d t\right]^{1 / 2},
$$

and note that from $\sqrt{3.26}$ we have that $|w|_{1,\left(I_{n} \times K\right)}^{2} \leq c\left(\eta_{\mathrm{lb}}^{n}\right)^{2}$. We then integrate by parts in 3.22 and use $w\left(\cdot, t^{i}\right)=0$ to obtain

$$
-\left(\left(\varepsilon, w_{t}\right)\right)_{\hat{G}^{n}}-\int_{t^{n}}^{t^{n+1}} \mathcal{B}(\varepsilon, w) d t-\left(\left(\widetilde{D} \varepsilon_{y}+\widetilde{E} \varepsilon_{\theta}+\beta_{1} \varepsilon, w\right)\right)_{\hat{G}^{n}}=\sum_{K \in T_{h}^{n}}\left(\eta_{\mathrm{lb}}^{n}\right)^{2} .
$$

Using (3.26) we arrive at

$$
\sum_{i=0}^{n-1} \sum_{K \in T_{h}^{i}}\left(\eta_{\mathrm{lb}}^{i}\right)^{2} \leq c \sum_{i=0}^{n-1} \sum_{K \in T_{h}^{i}}\left\{\|\varepsilon\|_{K}^{2}+\|\nabla \varepsilon\|_{K}^{2}\right\} .
$$

These estimates readlily provide lower bounds for the a posteriori estimate:

Theorem 3.7. The error $\varepsilon=\hat{u}-u_{h}$ satisfies

$$
\frac{1}{c} \sum_{i=0}^{n-1} \sum_{K \in T_{h}^{i}}\left(\eta_{\mathrm{lb}}^{i}\right)^{2} \leq \sum_{i=0}^{n-1} \sum_{K \in T_{h}^{i}}\left\{\|\varepsilon\|_{K}^{2}+\|\nabla \varepsilon\|_{K}^{2}\right\}
$$

where

$$
\left(\eta_{\mathrm{lb}}^{i}\right)^{2}:=\int_{t^{i}}^{t^{i+1}}\left\{|K|\left\|\Pi_{K}^{i}\left(A_{h}\right)\right\|_{L^{2}(K)}^{2}+\sum_{\ell \in E_{K}^{i}}|\ell|\left\|\Pi_{\ell}^{i}\left(B_{h}\right)\right\|_{L^{2}(\ell)}^{2}\right\} d t
$$


and

$$
\begin{aligned}
& A_{h}:=-\partial_{t} u_{h}+\hat{f}+A u_{h y y}+B u_{h y \theta}+C u_{h \theta \theta}+D u_{h y}+E u_{h \theta}+\beta_{1} u_{h}, \\
& B_{h}:=-\left[\mathcal{M} \nabla u_{h} \cdot \boldsymbol{n}\right]_{\ell} .
\end{aligned}
$$

We note that the lower bound, which is, of course, computable, is derived using projection to space-time constants. Furthermore, note that both Theorems 3.6 and 3.7 provide lower and upper a posteriori bounds, respectively, for the quantity

$$
\sum_{i=0}^{n-1} \sum_{K \in T_{h}^{i}}\left\{\|\varepsilon\|_{K}^{2}+\|\nabla \varepsilon\|_{K}^{2}\right\}
$$

\section{Numerical EXPERIMENTS}

We applied our method for the problem 1.2 in dimensions $m=2$ in space; recall that the space-time non-cylindrical domain $S_{T}$ has the form

$$
S_{T}=\{(x, t): x \in \Omega(t), 0<t<T\} .
$$

We considered the case where

$$
\Omega(t):=\left\{(z, \theta) \in \mathbb{R}^{2}: \quad z \in(0, s(t, \theta)), \quad \theta \in\left(\theta_{1}, \theta_{2}\right)\right\},
$$

for $t \in[0, T]$ and $s$ a smooth positive function. We took

$$
s(t, \theta)=1+0.25 \sin (\pi \theta) t, \quad 0 \leq t \leq 1, \quad 0<\theta<1,
$$

and defined

$$
u(z, \theta, t)=e^{t / 2} \frac{z \theta}{s^{2}(t, \theta)}\left(1-\frac{z}{s(t, \theta)}\right)^{3}\left(1-\frac{\theta}{s(t, \theta)}\right),
$$

as the exact solution of (1.2).

We applied the proposed transformation to obtain an equivalent problem of the form (2.16). The new initial and boundary value problem, now posed on a cylindrical space-time domain, was approximated by the discontinuous Galerkin scheme (3.3). This scheme was implemented in a double precision Fortran/C ++ code, where a uniform rectangular grid was used in space. To verify the theoretical result of Theorem 3.6 for the a posteriori upper bound, we computed the true error

$$
\mathcal{E}=\sum_{i=0}^{N-1} \sum_{K \in \mathcal{T}_{h}^{i}}\|\nabla \varepsilon\|_{K}^{2}+\sum_{i=0}^{N-1} \sum_{K \in \mathcal{T}_{h}^{i}}\|\varepsilon\|_{K}^{2}+\left|\varepsilon^{N}\right|_{\hat{\Omega}}^{2}+\sum_{i=0}^{N-1}\left|u_{h}^{i+0}-u_{h}^{i}\right|_{\hat{\Omega}}^{2},
$$

and the estimated error, $\mathcal{E}_{\text {est }}$, where we retained only the residual term and the 4 th, 5 th and 6 th terms appearing in the right-hand side of the estimate of Theorem 3.6. We took $q=3$ and used a range of space-time discretization parameters

$$
h=\max _{0 \leq n \leq N} h_{n} \quad k=\max _{0 \leq n \leq N} \boldsymbol{k}_{n} .
$$

We summarized our results in Table 1 below. The last column of Table 1 lists the effectivity index, computed as the ratio of the estimated error to the true error.

\section{APPENDIX}

This section presents some bounds concerning the local trace and local $L^{\infty}$ Sobolev inequalities, used throughout our proofs. Proofs are furnished for bounded, multi-dimensional domains with the constants depending on the domains' diameters, so that the results can be applied locally on each element of our partition. We begin by the trace inequality. 


\begin{tabular}{lllcr}
\hline Grid size & $h$ & $k$ & $\mathcal{E}$ & $\mathcal{E}_{\text {est }} / \mathcal{E}$ \\
\hline $10 \times 10$ & 0.1 & 0.025 & 0.631744 & 3.26 \\
$20 \times 20$ & 0.05 & 0.025 & 0.327328 & 2.18 \\
$40 \times 40$ & 0.025 & 0.025 & 0.174575 & 1.61 \\
$80 \times 80$ & 0.025 & 0.025 & 0.098069 & 1.94 \\
\hline $10 \times 10$ & 0.1 & 0.0125 & 0.425477 & 2.45 \\
$20 \times 20$ & 0.05 & 0.0125 & 0.216215 & 1.88 \\
$40 \times 40$ & 0.025 & 0.0125 & 0.108654 & 1.46 \\
$80 \times 80$ & 0.025 & 0.0125 & 0.054401 & 1.17 \\
\hline
\end{tabular}

TABle 1. Estimated and exact errors for problem 1.2 with $m=2$ and exact solution 4.1.

Theorem 5.1. Let $\mathcal{A}$ be a bounded domain in $\mathbb{R}^{n}$ with Lipschitz boundary. There exists a positive constant $c$, independent of $\mathcal{A}$, such that

$$
\|u\|_{L^{2}(\partial \mathcal{A})}^{2} \leq c\|u\|_{L^{2}(\mathcal{A})}\|u\|_{H^{1}(\mathcal{A})}+c(\operatorname{diam} \mathcal{A})^{-1}\|u\|_{L^{2}(\mathcal{A})}^{2}, \text { for any } u \in H^{1}(\mathcal{A}) .
$$

Proof. We use the general trace inequality (cf. [15])

$$
\|v\|_{L^{p}(\partial \hat{\mathcal{A}})} \leq c\|v\|_{L^{p}(\hat{\mathcal{A}})}^{1-1 / p}\|v\|_{W_{p}^{1}(\hat{\mathcal{A}})}^{1 / p}
$$

with $p=2$ and

so that

$$
\hat{\mathcal{A}}:=\left\{y \in R^{n}: \exists x \in \mathcal{A}: y=\frac{x}{\operatorname{diam} \mathcal{A}}\right\}
$$

$$
\|v\|_{L^{2}(\partial \hat{\mathcal{A}})}^{2} \leq c\|v\|_{L^{2}(\hat{\mathcal{A}})}\|v\|_{H^{1}(\hat{\mathcal{A}})},
$$

where $c$ is independent of $\hat{\mathcal{A}}$, since the measure of $\hat{\mathcal{A}}$ is of order $\mathcal{O}(1)$. In addition, using the change of variables $y=\frac{x}{\operatorname{diam\mathcal {A}}}, x \in \mathcal{A}$ and $\hat{u}(y)=u(x)$, iwe have, for any integer $k$, that

$$
|\hat{u}|_{H^{k}(\hat{\mathcal{A}})}=(\operatorname{diam} \mathcal{A})^{k-\frac{n}{2}}|u|_{H^{k}(\mathcal{A})} .
$$

Observing now the scaling for the the surface area $S_{n-1}$ of a ball in $\mathbb{R}^{n}$ of radius $\rho$, that is

$$
\int_{S_{n-1}} 1 d s=n c_{n} \rho^{n-1}
$$

we obtain, using (5.2),

$$
\begin{aligned}
\int_{\partial \mathcal{A}}|u|^{2} d s & \leq c(\operatorname{diam} \mathcal{A})^{n-1} \int_{\partial \hat{\mathcal{A}}}|\hat{u}|^{2} d s \leq c\|\hat{u}\|_{L^{2}(\hat{\mathcal{A}})}\|\hat{u}\|_{H^{1}(\hat{\mathcal{A}})}(\operatorname{diam} \mathcal{A})^{n-1} \\
& \leq c(\operatorname{diam} \mathcal{A})^{0-\frac{n}{2}}\|u\|_{L^{2}(\mathcal{A})}\left[(\operatorname{diam} \mathcal{A})^{1-\frac{n}{2}}|u|_{H^{1}(\mathcal{A})}+(\operatorname{diam} \mathcal{A})^{0-\frac{n}{2}}|u|_{L^{2}(\mathcal{A})}\right](\operatorname{dim} \mathcal{A})^{n-1} \\
& \leq c\|u\|_{L^{2}(\mathcal{A})}\|u\|_{H^{1}(\mathcal{A})}+c(\operatorname{diam} \mathcal{A})^{-1}\|u\|_{L^{2}(\mathcal{A})}^{2} .
\end{aligned}
$$

for some constant $c$, independent of $\mathcal{A}$.

The next result presents the $L^{\infty}$ Sobolev inequality for bounded domains of $\mathbb{R}^{n}$, for $n=1,2$, with constants depending on the diameter of the domain. The estimate for $n=2$ is not used in the proofs of this paper, but we include it for the sake of completeness. 
Theorem 5.2. Let $\mathcal{A}$ be a bounded domain in $\mathbb{R}^{n}, n=1,2$, with Lipschitz boundary, if $n=2$. Then, there exists a positive constant $c$, independent of $\mathcal{A}$, such that

$$
\|u\|_{L^{\infty}(\mathcal{A})}^{2} \leq c(\operatorname{diam} \mathcal{A})^{-1}\|u\|_{L^{2}(\mathcal{A})}^{2}+c(\operatorname{diam} \mathcal{A})|u|_{H^{1}(\mathcal{A})}^{2}, \quad \text { if } \quad n=1,
$$

and

$$
\|u\|_{L^{\infty}(\mathcal{A})}^{2} \leq c(\operatorname{diam} \mathcal{A})^{-2}\|u\|_{L^{2}(\mathcal{A})}^{2}+c|u|_{H^{1}(\mathcal{A})}^{2}+c(\operatorname{diam} \mathcal{A})^{2}|u|_{H^{2}(\mathcal{A})}^{2}, \quad \text { if } \quad n=2 .
$$

Proof. Using the notation of the previous theorem, we note first that

$$
\|u\|_{L^{\infty}(\mathcal{A})}=\|\hat{u}\|_{L^{\infty}(\hat{\mathcal{A}})} .
$$

If $n=1$, the Sobolev inequality and $(5.2)$ give 15$]$

$$
\begin{aligned}
\|\hat{u}\|_{L^{\infty}(\hat{\mathcal{A}})}^{2} & \leq c\|\hat{u}\|_{H^{1}(\hat{\mathcal{A}})}^{2}=c\|\hat{u}\|_{L^{2}(\hat{\mathcal{A}})}^{2}+c|\hat{u}|_{H^{1}(\hat{\mathcal{A}})}^{2} \\
& =c(\operatorname{diam} \mathcal{A})^{2(0-1 / 2)}\|u\|_{L^{2}(\mathcal{A})}^{2}+c(\operatorname{diam} \mathcal{A})^{2(1-1 / 2)}|u|_{H^{1}(\mathcal{A})}^{2},
\end{aligned}
$$

for some positive constant $c$ independent from $\mathcal{A}$. Hence, by $(5.5)$ and the above relation, the inequality (5.3) follows. Let us now consider the case $n=2$. The Sobolev inequality combined with 5.2 give

$$
\begin{aligned}
\|\hat{u}\|_{L^{\infty}(\hat{\mathcal{A}})}^{2} & \leq c\|\hat{u}\|_{H^{2}(\hat{\mathcal{A}})}^{2}=c\|\hat{u}\|_{L^{2}(\hat{\mathcal{A}})}^{2}+c|\hat{u}|_{H^{1}(\hat{\mathcal{A}})}^{2} \\
& =c(\operatorname{diam} \mathcal{A})^{2(0-2 / 2)}\|u\|_{L^{2}(\mathcal{A})}^{2}+c(\operatorname{diam} \mathcal{A})^{2(1-2 / 2)}|u|_{H^{1}(\mathcal{A})}^{2}+c(\operatorname{diam} \mathcal{A})^{2(2-2 / 2)}|u|_{H^{2}(\mathcal{A})}^{2},
\end{aligned}
$$

so (5.4) follows easily by using the above relation and 5.5 .

\section{ACKNOWLEDGMENTS}

The authors would like to thank Prof. Ch. Makridakis for stimulating discussions, and the two anonymous referees for their valuable comments.

\section{REFERENCES}

[1] G. Akrivis, V.A. Dougalis, G.E. Zouraris, Finite Difference Schemes for the 'Parabolic' Equation in a variable depth environment with a rigid bottom boundary condition, SIAM J. Numer. Anal. 39, 539-565 (2001)

[2] G. Akrivis, C. Makridakis, Galerkin time-stepping methods for nonlinear parabolic equations. ESAIM: Math. Mod. and Num. Anal. 38, 261-289 (2004)

[3] R. Alexander, Diagonally Implicit Runge-Kutta Methods for stiff O.D.E.'S. SINUM 14(6), 1006-1021 (1977)

[4] D.C. Antonopoulou, Theory and Numerical Analysis of Parabolic Approximations, Ph.D. Thesis, Department of Mathematics, University of Athens, Greece, 2006

[5] D.C. Antonopoulou, V.A. Dougalis, G.E. Zouraris, Galerkin methods for Parabolic and Schrödinger Equations with dynamical boundary conditions and applications to underwater acoustics, SIAM J. Numer. Anal. 47(4), 2752-2781 (2009)

[6] D.C. Antonopoulou, G.D. Karali, M. Plexousakis, G.E. Zouraris, Crank-Nicolson Finite Element Discretizations for a 2D Linear Schrödinger-Type Equation Posed in a Noncylindrical Domain, Math. Comp., 84(294), 1571-1598 (2015)

[7] D.C. Antonopoulou, M. Plexousakis, Discontinuous Galerkin methods for the linear Schrödinger equation in non-cylindrical domains, Numer. Math. 115, 585-608 (2010)

[8] I. Babuska, R. Duran, R. Rodriguez, Analysis of the efficiency of an a posteriori error estimator for linear triangular finite elements, SIAM J. Numer. Anal. 29(4), 947-964 (1992)

[9] M. J. Baines. Moving finite elements, Monographs on Numerical Analysis. The Clarendon Press, Oxford University Press, New York, 1994, Oxford Science Publications.

[10] J. Baranger, H. El-Amri, Estimateurs a posteriori d' erreur pour le calcul adaptif d' écoulements quasi-newtoniens, RAIRO M2AN. 25(1), 31-48 (1991)

[11] C. Bernardi, Optimal finite element interpolation on curved domains, SIAM J. Numer. Anal. 26(5), 1212-1240 (1989)

[12] K.S. Bey, A. Patra, J.T. Oden, hp-version discontinuous Galerkin methods for hyperbolic conservation laws: A parallel strategy, Internat. J. Numer. Methods Engrg. 38, 3889-3908 (1995)

[13] K.S. Bey, A. Patra, J.T. Oden, hp-version discontinuous Galerkin methods for hyperbolic conservation laws. Comput. Methods Appl. Mech. Engrg. 133, 259-286 (1996)

[14] K.S. Bey, A. Patra, J.T. Oden, A parallel hp-adaptive discontinuous Galerkin method for hyperbolic conservation laws. Appl. Numer. Math. 20, 321-386 (1996)

[15] S. C. Brenner, L. Ridgway Scott, The Mathematical Theory of Finite Element Methods. Springer-Verlag, New York, 1991

[16] P. Clément, Approximation by finite element functions using local regularization, RAIRO Anal. Numér. 9, 77-84 (1975) 
[17] B. Cockburn, C.W. Shu, The local discontinuous Galerkin method for time-dependent convection-diffusion systems. SIAM J. Numer. Anal. 35 no. 6, 2440-2463 (1998)

[18] M. Crouzeix, Sur l'approximation des eq́uations diffeŕentielles opeŕationnelles lineáires par des met́hodes de Runge Kutta, Thes̀e, University Paris VI, Paris, 1975.

[19] L.C. Evans, Partial Differential Equations. American Mathematical Society, 1998

[20] G. Fichera, On a unified theory of boundary value problems for elliptic - parabolic equations of second order, Boundary Problems in Differential Equations, University of Wisconsin Press, Madison, WI, 1960.

[21] A. Hiltebrand, S. Mishra, Entropy stable shock capturing space-time discontinuous Galerkin schemes for systems of Conservation Laws. Research Report No. 2012-07, ETHZ, 2012

[22] A. Hiltebrand, S. Mishra, C. Parés, Entropy-stable space-time DG schemes for non-conservative hyperbolic systems. ESAIM: M2AN, 2017, DOI: https://doi.org/10.1051/m2an/2017056

[23] P. Jamet, Numerical methods and existence theorems for parabolic differential equations whose coefficients are singular on the boundary. Math. Comput. 22, 721-743 (1968)

[24] P. Jamet, Estimation d'erreur pour des éleménts finis droits presque dégénérés, Rev. Francaise Automat. Informat. Recherche Opérationelle Sér. Rouge Anal. Numér. 10, 43-61 (1976)

[25] P. Jamet, Galerkin-type approximations which are discontinuous in time for parabolic equations in a variable domain. SIAM J. Numer. Anal. 15, 913-928 (1978)

[26] O. Karakashian, C. Makridakis, A space-time finite element method for the Nonlinear Schrödinger Equation: The Discontinuous Galerkin Method. Math. Comp. 67, 479-499 (1998)

[27] O.A. Karakashian and F. Pascal, A posteriori error estimates for a Discontinuous Galerkin approximation of second-order Elliptic problems, SIAM J. Numer. Anal. 41(6), 2374-2399 (2003)

[28] C. Klaij, J. J.W. V. der Vegt, H. V. der Ven, Space-time discontinuous Galerkin method for the compressible Navier-Stokes equations. Journal of Comp. Physics 217, 589-611 (2006)

[29] P. Lesaint, P.A. Raviart, On a finite element method for solving the neutron transport equation. Mathematical Aspects of Finite Elements in Partial Differential Equations (C. deBoor, ed.), Academic Press, New York (1974)

[30] J. L. Lions, Sur les problemes mixtes pour certains systemes paraboliques dans des ouverts non cylindriques. Ann. Inst. Fourier 7, 143-182 (1957)

[31] C. Makridakis and R. H. Nochetto, A posteriori error analysis for higher order dissipative methods for evolution problems, Numer. Math. 104(4), 489-514 (2006)

[32] G. May, M. Zakerzadeh, On the Convergence of Space-Time Discontinuous Galerkin Schemes for Scalar Conservation Laws. SIAM J. Numer. Anal., 54(4), 2452-2465 (2016)

[33] A. L. Mignot, Methodes d'approximation des solutions de certains problemes aux limites lineaires, Rend. Sem. Mat. Univ. Padova, XL (1968)

[34] K. Miller, Moving Finite Elements, II. SINUM 18(6), 1033-1057 (1981)

[35] L. Pareschi, G. Russo, Implicit-explicit Runge-Kutta schemes and applications to hyperbolic systems with relaxation. Journal of Scientific Computing 25, 129-155 (2005)

[36] M. Picasso, Adaptive finite elements for a linear parabolic problem. Comput. Meth. Appl. Mech. Engrg. 167, 223-237 (1998)

[37] W.H. Reed, T.R. Hill, Triangular mesh methods for the neutron transport equation. Tech. Report LA-UR-73-479, Los Alamos Scientific Laboratory (1973)

[38] D. Schötzau and T.P. Wihler, A posteriori error estimation for hp-version time-stepping methods for parabolic partial differential equations, Numer. Math. 115(3), 475-509 (2010)

[39] M. Tavelli, M. Dumbser, A staggered space-time discontinuous Galerkin method for the three-dimensional incompressible Navier-Stokes equations on unstructured tetrahedral meshes. J. Comput. Phys. 319, 294-323 (2016).

[40] M. Tavelli, M. Dumbser, Arbitrary high order accurate space-time discontinuous Galerkin finite element schemes on staggered unstructured meshes for linear elasticity, arXiv:1802.04516v1 (2018)

[41] J. J. W. van der Vegt, H. van der Ven, Space-time discontinuous Galerkin finite element method with dynamic grid motion for inviscid compressible flows I. general formulation, Journal of Comp. Phys. 182, 546-585 (2002)

[42] H. van der Ven, J. J. W. van der Vegt, Space-time discontinuous Galerkin finite element method with dynamic grid motion for inviscid compressible flows II. efficient flux quadrature, Comput. Methods Appl. Mech. Engrg. 191, 4747-4780 (2002)

[43] R. Verfürth, A posteriori error estimators for the Stokes equations, Numer. Math. 55, 309-325 (1989) 